\title{
Exponential Decay and Geometric Aspect of Transition Probabilities in the Adiabatic Limit
}

\author{
A. JOYE AND H. KunZ \\ Department of Physics, Ecole Polytechnique Fédérale de Lausanne, \\ CH-1015 Lausanne, Switzerland \\ AND \\ Ch.-Ed. Pfister \\ Department of Mathematics, Ecole Polytechnique Fédérale de Lausanne, \\ CH-1015 Lausanne, Switzerland
}

Received August 10, 1990; revised December 19, 1990

\begin{abstract}
We consider a quantum mechanical system whose hamiltonian is a time-dependent analytic $n \times n$ matrix. For $n=2$ we establish a generalization of Dykhne formula which gives the transition probability from one energy level to the other in the adiabatic limit. We discuss in particular the geometric nature of this formula. In the general case, $n \geqslant 2$, we prove an upper bound for the probability of such transitions which shows that they are exponentially small. 1991 Academic Press, Inc.
\end{abstract}

\section{INTRODUCTION}

The Adiabatic Theorem of quantum mechanics has a long history. It has already been studied by Born and Fock [1]. The theorem describes the asymptotic regime of the slow evolution of a quantum mechanical system. This is an important limit which is discussed in many textbooks on quantum mechanics. Recently, a lot of work has been done on the topological or geometric aspect of the theorem after the publication of a paper by Berry [2] (see the collection of papers [3]). The geometric interpretation of the results of [2] has been clearly exposed by Simon in [4].

We consider a quantum mechanical system whose evolution is governed by the Schroedinger equation $(h=1)$

$$
i \frac{d \psi}{d t^{\prime}}\left(t^{\prime}\right)=H\left(t^{\prime} / T\right) \psi\left(t^{\prime}\right) .
$$

The parameter $T$ is the typical time-scale over which the hamiltonian changes 
a finite amount. Introducing the rescaled time $t=t^{\prime} / T$ we obtain the equivalent equation

$$
i \frac{d \psi_{T}}{d t}(t)=T H(t) \psi_{T}(t)
$$

The adiabatic limit corresponds to the limit $T \rightarrow \infty$. In this limit, if the system at time $t_{0}$ is in the ground state of $H\left(t_{0}\right)$, then at time $t$ it is in the ground state of $H(t)$ provided that the ground states of $H(s), t_{0} \leqslant s \leqslant t$, are isolated in the spectrum of $H(s)$. A natural and important question is to estimate the probability of transition to an excited state when $T$ is large. It is believed that analyticity of the hamiltonian leads to exponentially small transition probabilities under appropriate conditions. This is this problem which we study here. Throughout the paper we make the following two assumptions

I. Analyticity. There exists a simply connected domain $S_{u}$ with the following properties: the hamiltonian $H(t), t \in \mathbb{R}$ is a $n \times n$ hermitian matrix which has an analytic extension on $S_{a} ; S_{a}=\overline{S_{a}}$ and $\exists a$ such that $\forall z=t+i s,|s|<a$ and $|t|$ large enough, $z \in S_{a}$.

II. Behaviour at infinity. There exist two $n \times n$ hermitian matrices $H^{+}$and $H^{-*}$ such that

$$
\lim _{t \rightarrow \pm \infty} \sup _{|s|<a}\left\|H(t+i s)-H^{ \pm}\right\||t|^{1+\varepsilon}=0
$$

for some positive $\varepsilon$. Moreover, the spectra of $H^{+}$and $H^{-}$are nondegenerate.

In physics many interesting phenomena are studied with the help of $n$-level systems and in particular 2-level systems. See, for example, the recent review paper by Solov'ev [5] in which the problem of computation of transition probabilitics in the adiabatic limit (called nonadiabatic transitions) is treated. This problem leads to the study of global properties of an analytic function. This is mainly the aspect which we consider here, since it has been neglected in the literature. Because of this fact, incorrect conclusions have been drawn (see below the discussion of formula (1.6) and comments on example (2.1)).

We first deal with the 2-level systems under the additional assumption

III. Separation of the spectrum. For each $t \in \mathbb{R}$ the spectrum of $H(t)$ consists of two separated eigenvalues $e_{1}(t)$ and $e_{2}(t)$ such that $e_{2}(t)-e_{1}(t) \geqslant \delta>0$.

We denote by $P_{1}(t)$ and $P_{2}(t)$ the spectral projections associated with the eigenvalues $e_{1}(t)$ and $e_{2}(t)$. By assumption II, $e_{i}(t)$ and $P_{i}(t)$ have well-defined limits as $t \rightarrow \pm \infty, i=1,2$. The basic question which we study is formulated as follows. Let $\psi_{T}(t)$ be a solution of the Schroedinger equation (1.2) which is normalized, $\left\|\psi_{T}(t)\right\|=1$, and which satisfies the boundary condition

$$
\lim _{t \rightarrow-\infty}\left\|P_{1}(t) \psi_{T}(t)\right\|=1 .
$$


This boundary condition means that the system is prepared at time $t--\infty$ so that its energy is $e_{1}(-\infty)$ with probability one. We let the system evolve and measure its energy at some later time $t$. The result of this measurement is either $e_{1}(t)$ or $e_{2}(t)$. Since we have separation of the spectrum for all $t$ the probability that we find the value $e_{2}(t)$ is

$$
\left\|P_{2}(t) \psi_{I}(t)\right\|^{2} \leqslant c / T^{2},
$$

where $c$ is a constant independent of $t$. Thus, when $T \rightarrow \infty$, the probability that the system has made a transition to the encrgy level $e_{2}(t)$ tends to zero. This is the content of the Adiabatic Theorem (The analyticity assumption is not needed for the validity of the bound (1.4)).

Ihe situation is quite different if we perform the measurement of the energy at time $t=+\infty$. Let us suppose that there are two eigenvalue crossings at $z_{1}$ and $\bar{z}_{1}$ in $S_{a}$ (see Fig. (1)). We also suppose that the eigenvalues $e_{i}(z)$ and eigenprojections $P_{i}(z)$ have an algebraic singularity of square root type at $z_{1}$ and $\bar{z}_{1}$. Let $x_{0}$ be any point of the real axis. In a neighbourhood of $x_{0}$ the eigenvalues are analytic and we can choose analytic eigenvectors $\varphi_{i}(z), H(z) \varphi_{i}(z)=e_{i}(z) \varphi_{i}(z)$, so that $\varphi_{1}\left(x_{0}\right)$ and $\varphi_{2}\left(x_{0}\right)$ form an orthonormal basis. Let $\gamma$ be a loop as in Fig. 1. We analytically continue $e_{1}(z)$ and $\varphi_{1}(z)$ along the path $\gamma$ and we denote by $\tilde{e}_{1}\left(x_{0}\right), \widetilde{\varphi}_{1}\left(x_{0}\right)$ the result of the analytic continuation when we come back to $x_{0}$. We have

$$
\tilde{e}_{1}\left(x_{0}\right)=e_{2}\left(x_{0}\right), \quad \tilde{\rho}_{1}\left(x_{0}\right)=e^{-i t_{21}\left(x_{0}|r| r\right.} \varphi_{2}\left(x_{0}\right)
$$

where $\theta_{21}\left(x_{0} \mid \gamma\right)$ is in general a complex phase. We also define $\int_{;} e_{1}$ as the integral of the analytic continuation of $e_{1}$ along $\gamma$. Both quantities $\theta_{21}\left(x_{0} \mid \gamma\right)$ and $\int_{\gamma_{1}} e_{1}$ depend only on the homotopy class of the loop $\gamma$ starting and ending at $x_{0}$. Moreover, $\operatorname{Im} \theta_{21}\left(x_{0} \mid \gamma\right)$ and $\operatorname{Im} \int_{i_{1}} e_{1}$ do not depend on the choice of $x_{0}$ as long as $x_{0} \in \mathbb{R}$. Under an additional hypothesis, which is introduced in Section 2.3 (condition VI) and analyzed in Section 2.4 , the probability that we measure the energy $e_{2}(+\infty)$ at time $t=+\infty$ is given by

$\lim _{t \rightarrow \infty}\left\|P_{2}(t) \psi_{T}(t)\right\|^{2}=\exp \left(2 \operatorname{Im} \theta_{21}\left(x_{0} \mid \gamma\right)\right) \exp \left(2 T \operatorname{Im} \int_{\gamma} e_{1}\right)\left(1+O\left(\frac{1}{T}\right)\right)$.
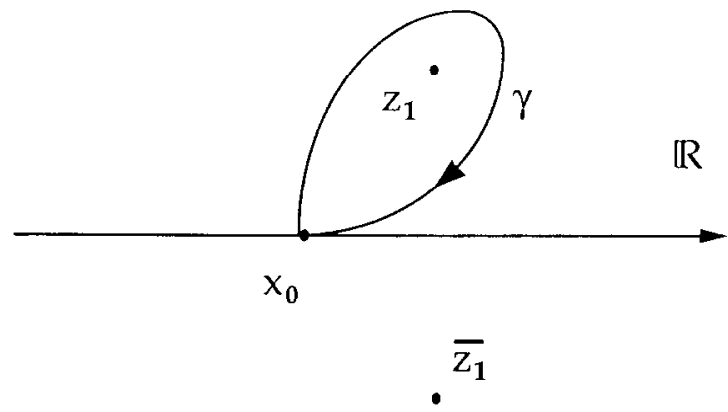

FIG. 1. Eigenvalue-crossings in $S_{u}$. 
Since we can choose any $x_{0} \in \mathbb{R}$ we can take $x_{0}-\operatorname{Re} z_{1}$. Then

$$
\operatorname{Im} \int_{\gamma} e_{1}=\int_{0}^{\operatorname{Im}=1} \operatorname{Re}\left(e_{1}\left(x_{0}+i s\right)-e_{2}\left(x_{0}+i s\right)\right) d s .
$$

Thus, provided (1.6) is correct, the probability that we measure $e_{2}(+\infty)$ is exponentially small in $T$. Formula (1.6) with $\exp \left(2 \operatorname{Im} \theta_{21}\left(x_{0} \mid \gamma\right)\right)$ replaced by 1 , is known as Dykhne formula [6] and can be found in Landau and Lifschitz [7]. A convincing derivation of it has been given in 1977 in a very interesting paper of Hwang and Pechukas [8], but without the factor $\exp \left(2 \operatorname{Im} \theta_{21}\left(x_{0} \mid \gamma\right)\right)$. In Section 2, we again prove formula (1.6), using the ideas of Hwang and Pechukas.

We add new results to their paper. First, there is the geometric factor $\exp \left(2 \operatorname{Im} \theta_{21}\left(x_{0} \mid \gamma\right)\right)$ in (1.6). The phase $\theta_{21}\left(x_{0} \mid \gamma\right)$ is the analog of the Berry phase for a loop in the complex plane around the eigenvalue-crossing $z_{1}$. However, this does not correspond to a loop in the space of hamiltonians. Second, the proof of formula (1.6) by the method of [8] is valid only if we make an additional hypothesis on the global behaviour of the analytic continuation of the function

$$
\int_{x_{0}}^{z}\left(e_{1}\left(z^{\prime}\right)-e_{2}\left(z^{\prime}\right)\right) d z^{\prime}
$$

The discussion of this hypothesis in Section 2.4 is quite important, although it appears as a technical hypothesis in the derivation of (1.6). Indeed, let us suppose that we do not have two, but more, eigenvalue-crossings in $S_{a}$, say $z_{1}$ and $\overline{z_{1}}, z_{2}$, and $\overline{z_{2}}$. Which eigenvalue-crossing must be used in order to compute the transition probability using formula (1.6)? This is obviously an important issue since the result given by (1.6) depends on this choice. It could seem that a natural choice is to consider the closest eigenvalue-crossing to the real axis (implicitly in the Euclidean distance) as in $[8,9]$. However, if the global character of the problem is taken into account then it turns out that this is clearly not so. Indeed the analysis of Section 2.4 shows that there is a metric $d_{p}$, constructed with the eigenvalues of the hamiltonian $H$ such that whenever we can prove (1.6) with the method of Section 2.3 the eigenvalue-crossing which governs formula (1.6), say $z_{1}$, is the nearest eigenvalue-crossing to the real axis in this metric $d_{p}$. An explicit example where this happens is given (Example (2.1)). Moreover, (1.6) can be stated in a purely geometric way,

$$
\lim _{t \rightarrow \sim}\left\|P_{2}(t) \psi_{T}(t)\right\|^{2}=\exp \left(2 \operatorname{Im} \theta_{21}\left(x_{0} \mid \gamma\right)\right) \exp \left(-2 T \operatorname{dist}_{\rho}\left(z_{1}, \mathbb{R}\right)\right)\left(1+O\left(\frac{1}{T}\right)\right) .
$$

The simplicity of (1.9) is only apparent. In particular, we must know some global properties of the analytical continuation of the eigenvalues in order to compute $\operatorname{dist}_{\rho}\left(z_{1}, \mathbb{R}\right)$. On the other hand, it seems that this metric gives the correct notion of distance. This is confirmed by an example of Section 2.4, where the hamiltonian 
has a singularity which is closer to the real axis in the Euclidean distance than the eigenvalue-crossing. However, in terms of the metric $d_{\rho}$ the converse is true, and we can show that formula (1.6) is still true. The introduction of the metric $d_{\rho}$ as well as formula (1.9) is a new result in the subject, although this metric has been used for a long time in the theory of Teichmueller spaces.

From the above discussion it is quite clear that it is welcome to have upper bounds for $\lim _{t \rightarrow \infty}\left\|P_{2}(t) \psi_{T}(t)\right\|^{2}$ without additional hypothesis. This question is solved in Section 3, not only for two-level systems, but in the more general case of a $n$-level system. There are few results for this general case. Some aspects are treated by Solov'ev [5]. The paper [8] also contains a theorem for the $n$-level case; however, in general, the hypotheses of this theorem are not fulfilled (see Section 3.2). We can obtain rigorous exponential bounds for any $n \times n$ matrix $H(z)$ which satisfies conditions I, II, and IV.

IV. Existence of an isolated energy level. There exists on the real axis one energy level, say $e_{k}(t)$, which is always separated from the other energy levels $e_{i}(t)$ :

$$
\left|e_{k}(t)-e_{i}(t)\right| \geqslant \delta>0, \quad i \neq k, \forall t \in \mathbb{R} .
$$

Let $P_{i}(t)$ denote the spectral projection associated with the energy $e_{i}(t)$, and let $\psi_{T}(t)$ be a solution of Eq. (1.2) such that $\left\|\psi_{T}(t)\right\|=1$ and

$$
\lim _{t \rightarrow \cdots x}\left\|P_{k}(t) \psi_{T}(t)\right\|=1
$$

Then the probability that we measure at time $t=+\infty$ the energy $e_{i}(+\infty), i \neq k$, is exponentially small,

$$
\lim _{t \rightarrow+\infty}\left\|P_{i}(t) \psi_{T}(t)\right\|^{2} \leqslant \frac{M}{T^{2}} \exp \left(-k T\left|e_{i}(+\infty)-e_{k}(+\infty)\right|\right),
$$

where $M$ and $\kappa$ are two positive constants. The constant $\kappa$ is given in (3.23).

Whenever we can prove (1.6) for $n=2$, we can show that the best upper bound obtained by the method of Section 3 gives the correct behaviour in $T$ for the transition probability: the best upper bound is equal to

$$
\lim _{r \rightarrow+\infty}-\frac{1}{T} \ln \left(\lim _{s \rightarrow+\infty}\left\|P_{2}(t) \psi_{T}(t)\right\|^{2}\right)=\left|e_{2}(+\infty)-e_{1}(+\infty)\right| \sup _{\kappa}\{\kappa \text { as in }(1.11)\} .
$$

Comments. Some of these results, in particular (1.6) have been announced in [10]. During the completion of this paper we received a preprint of M. Berry [9], where he points out independently of us that formula (1.6) must contain the geometric factor $\exp \left(2 \operatorname{Im} \theta_{21}\left(x_{0} \mid \gamma\right)\right)$. We also mention that the results of Section 3 have been generalized to the case of unbounded operators [11]. We thank M. Berry for having communicated his results to us. 


\section{Spin- $\frac{1}{2}$ In $\wedge$ Time-Dependent Magnetic Field}

\subsection{Coordinate-Dependent Formulation of the Problem}

Let $H(z)$ be a $2 \times 2$ matrix which satisfies conditions I, II, and III of Section 1 in $S_{a}$. Without restricting the generality we suppose that the trace of $H(z)$ is identically zero, so that

$$
\begin{aligned}
H(z) & =\mathbf{B}(z) \cdot \boldsymbol{\sigma} \\
& \equiv B_{1}(z) \frac{1}{2}\left(\begin{array}{ll}
0 & 1 \\
1 & 0
\end{array}\right)+B_{2}(z) \frac{1}{2}\left(\begin{array}{cc}
0 & -i \\
i & 0
\end{array}\right)+B_{3}(z) \frac{1}{2}\left(\begin{array}{rr}
1 & 0 \\
0 & -1
\end{array}\right) .
\end{aligned}
$$

The functions $B_{k}$ are analytic in $S_{a}, B_{k}(\bar{z})=\overline{B_{k}(z)}$, and $\lim _{t \rightarrow \pm x} B_{k}(t)=B_{k}( \pm)$ exist $(t \in \mathbb{R})$. Moreover, for some positive $\varepsilon$,

$$
\lim _{t \rightarrow \pm x} \sup _{|s|<a}\left|B_{k}(t+i s)-B_{k}( \pm)\right||t|^{1+\varepsilon}=0 .
$$

The hamiltonian (2.1) is interpreted as the hamiltonian of a spin- $\frac{1}{2}$ in a magnetic field B. Let $\rho(z)=B_{1}^{2}(z)+B_{2}^{2}(z)+B_{3}^{2}(z)$. The eigenvalues of $H(z)$ are $\pm \frac{1}{2} \sqrt{\rho(z)}$, where $\sqrt{ }$ is the branch of the square root which takes the value 1 at $z=1$. Condition III implies that $\rho(t)>0$ for $t \in \mathbb{R}$. We define

$$
e_{1}(t)=-\frac{1}{2} \sqrt{\rho(t)}, \quad e_{2}(t)=\frac{1}{2} \sqrt{\rho(t)} .
$$

The corresponding eigenprojections are

$$
P_{k}(t)=\frac{1}{2}\left(\square+(-1)^{k} \frac{\mathbf{B}(t) \cdot \boldsymbol{\sigma}}{(1 / 2) \sqrt{\rho(t)}}\right), \quad k=1,2 .
$$

Let $\psi_{T}(t)$ be a normalized solution of the Schroedinger equation

$$
i \frac{d}{d t} \psi_{T}(t)=T H(t) \psi_{T}(t)
$$

with the property

$$
\lim _{t \rightarrow \infty}\left\|P_{1}(t) \psi_{T}(t)\right\|=1
$$

We want to study the behaviour of such a solution at $t=+\infty$. To simplify the problem we introduce the supplementary condition $V$. This condition will be removed in Section 2.4 .

V. Eigenvalue-crossings. The set $X$ of zeros of $\rho(z)$ in $S_{a}$ consists of exactly two points $z_{1}$ and $\overline{z_{1}}$ and these points are simple zeros of $\rho(z)$.

The condition means that there are exactly two eigenvalue-crossings in $S_{a}$, located at $z_{1}$ and $z_{1}$, and that each of these points is a branching point of order 1 for the eigenvalues and eigenprojections. 
The solution $\psi_{T}(t)$ has an analytic extension on $S_{a}, \psi_{T}(z)$, which is a singlevalued function satisfying the differential equation

$$
i \psi_{T}^{\prime}(z)=T H(z) \psi_{T}(z)
$$

Here, and throughout the paper, ' denotes $d / d z, z$ complex. In order to study the solution $\psi_{T}(z)$ we decompose $\psi_{T}(z)$ in a $z$-dependent basis of eigenvectors of $H(z)$, $\varphi_{1}(z)$ and $\varphi_{2}(z)$, so that

$$
H(z) \varphi_{k}(z)=e_{k}(z) \varphi_{k}(z)
$$

and we require that $\varphi_{k}(z)$ are analytic. There are several ways to do this because $e_{k}$ and $P_{k}$ have analytic extensions on $M=S_{a} \backslash X$ which are multi-valued. We use this fact in an essential way in the study of $\psi_{T}$. However, for the moment, let $\Omega$ be the simply-connected domain of $S_{a}$ which is obtained by removing two lines issued at $z_{1}$ and $\bar{z}_{1}$ and joining the boundary of $S_{a}$ (see Fig. 2 ). Since $\Omega$ is simply-connected, the eigenvalues $e_{k}(t)$ have a single-valued analytic extension $e_{k}(z)$ on $\Omega$. The same is true for $P_{k}(z)$. These analytic extensions are explicitly given by the analytic continuations of formulas (2.3) and (2.4). We construct an analytic family of z-dependent basis on $\Omega$ using a standard method of the theory of perturbations. For details, see Kato [12, Chap. II.4]. We introduce the operator

$$
K(z)-P_{1}^{\prime}(z) P_{1}(z)+P_{2}^{\prime}(z) P_{2}(z)
$$

which is given explicitly by

$$
K(z)=i \frac{\mathbf{B}(z) \wedge \mathbf{B}^{\prime}(z)}{\rho(z)} \cdot \boldsymbol{\sigma},
$$

where $\mathbf{B} \wedge \mathbf{B}^{\prime}$ is the vector product of $\mathbf{B}$ and $\mathbf{B}^{\prime}$. On $\Omega$ we consider the solution $U(z)$ of

$$
U^{\prime}(z)=K(z) U(z), \quad U(0)=\rrbracket
$$

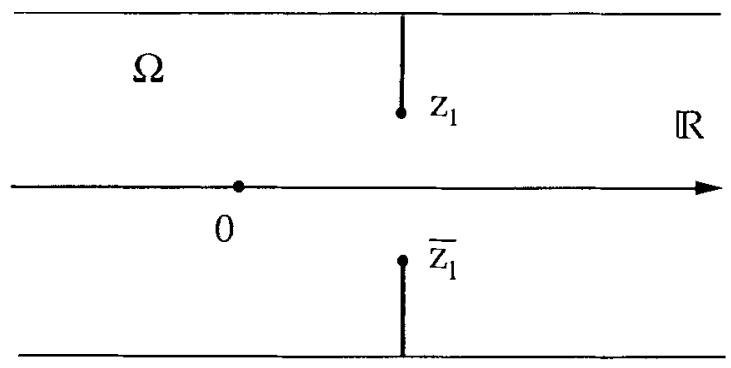

Fig. 2. The domain $\Omega$. 
with initial condition at 0 . (It is convenient to choose the initial condition on the real axis). We choose a basis of normalized eigenvectors $\varphi_{1}(0)$ and $\varphi_{2}(0)$,

$$
H(0) \varphi_{k}(0)=e_{k}(0) \varphi_{k}(0), \quad k=1,2 .
$$

Using the operator $U(z)$ we define

$$
\varphi_{k}(z)=U(z) \varphi_{k}(0), \quad k=1,2 .
$$

This gives a choice of analytic eigenvectors of $H(z)$. This follows from the relation

$$
U(z) P_{k}(0)=P_{k}(z) U(z), \quad k=1,2,
$$

so that

$$
P_{k}(z) \varphi_{k}(z)=\varphi_{k}(z), \quad k=1,2 .
$$

The eigenvectors $\varphi_{k}(z), k=1,2$, have the property

$$
P_{k}(z) \varphi_{k}^{\prime}(z)=0, \quad k=1,2 .
$$

Indeed, using (2.13) and (2.9)

$$
P_{k}(z) \varphi_{k}^{\prime}(z)=P_{k}(z)\left(P_{1}^{\prime}(z) P_{1}(z)+P_{2}^{\prime}(z) P_{2}(z)\right) \varphi_{k}(z)=0,
$$

since

$$
P_{k}(z) P_{k}^{\prime}(z) P_{k}(z)=0 .
$$

Notice also that $U(z)$ has limits as $\operatorname{Re} z \rightarrow \pm \infty$ and $|\operatorname{Im} z|<a$. This is a consequence of condition II. This condition implies that for large $|t|$,

$$
\sup _{|s|<a}\|K(t+i s)\| \equiv k(t)
$$

is an integrable function of $t$. The operator $U(z)$ is solution of the Volterra equation on $\Omega$,

$$
U(z)=\rrbracket+\int_{0}^{z} K\left(z^{\prime}\right) U\left(z^{\prime}\right) d z^{\prime},
$$

where in (2.20) the integral is over any path in $\Omega$ from 0 to $z$. This equation can be solved by iteration and from the integrability of the function $k(t)$ it is not difficult to show that there exist operators $U(+)$ and $U(-)$ such that

$$
\lim _{t \rightarrow \pm s:} \sup _{|s|<a}\|U(t+i s)-U( \pm)\|=0 .
$$

Let

$$
\lambda_{k}(z)=\int_{0}^{z} e_{k}\left(z^{\prime}\right) d z^{\prime}, \quad k=1,2
$$


and

$$
\Delta_{i j}(z)=i_{i}(z)-\lambda_{j}(z), \quad i \neq j
$$

where in (2.22) the integral is over any path in $\Omega$ starting at 0 and ending at $z$. We write

$$
\psi_{T}(z)=\sum_{j=1}^{2} c_{j}(z) e^{-i T \lambda_{1}(z)} \varphi_{j}(z)
$$

and look for a differential equation for the coefficients $c_{j}(z)$. From Eq. (2.7), we obtain

$$
\sum_{j=1}^{2}\left(c_{j}^{\prime}(z) e^{i T i j(z)} \varphi_{j}(z)+c_{j}(z) e^{-i T \lambda_{j}(z)} \varphi_{j}^{\prime}(z)\right)=0 .
$$

Taking the scalar product of the expression (2.25) with the vector $\left(U(z)^{1}\right)^{*} \varphi_{k}(0)$ we obtain, using (2.14) and (2.16),

$$
c_{k}^{\prime}(z)=\sum_{j=1}^{2} a_{k j}(z) e^{i T \cdot s_{k j} l=1} c_{j}(z), \quad j \neq k
$$

with

$$
\begin{aligned}
a_{k j}(z) & =-\left\langle\varphi_{k}(0) \mid U(z)^{-1} \varphi_{j}^{\prime}(z)\right\rangle \\
& -\left\langle\varphi_{k}(0) \mid U(z)^{-1} K(z) U(z) \varphi_{j}(0)\right\rangle
\end{aligned}
$$

If $|\operatorname{Im} z|<a$ and $|\operatorname{Re} z|$ is large enough then

$$
\left|a_{k j}(z)\right| \leqslant C \cdot k(t), \quad z=t+i s
$$

for some constant $C$. Although $\psi_{T}(z)$ does not have a limit as $\operatorname{Re} z \rightarrow \pm \infty$, because of the presence of the phases $\lambda_{k}(z)$, the coefficients $c_{k}(z)$ and the vectors $\varphi_{k}(z)$ have well-defined limits. In particular the boundary condition $(2.6)$ for normalized $\psi_{T}(z)$ reads

$$
\begin{aligned}
& \lim _{x}\left|c_{1}(t)\right|=\left|c_{1}(-\infty)\right|=1 \\
& \lim _{t \rightarrow-\infty}\left|c_{2}(t)\right|=\left|c_{2}(-\infty)\right|=0 .
\end{aligned}
$$

Our problem becomes equivalent to the determination of the coefficients $c_{1}(+\infty)$ and $c_{2}(+\infty)$. The coefficients $c_{k}(z)$ are analytic functions on $\Omega$. They satisfy Eq. (2.26) or the Volterra equation

$$
c_{1}(z)=c_{1}\left(z_{0}\right)+\int^{z} a_{12}\left(z^{\prime}\right) e^{i T \cdot 1_{12}\left(r^{\prime}\right)} c_{2}\left(z^{\prime}\right) d z^{\prime}
$$


and

$$
c_{2}(z)=c_{2}\left(z_{0}\right)+\int_{z_{0}}^{z} a_{21}\left(z^{\prime}\right) e^{i T 1_{21}\left(z^{\prime}\right)} c_{1}\left(z^{\prime}\right) d z^{\prime}
$$

where the integration is over any path in $\Omega$ starting at $z_{0}$ and ending at $z$. Explicitly, if $u \mapsto \gamma(u)$ is a path with $\gamma\left(u_{0}\right)=z_{0}$ and $\gamma(u)=z$, then

$$
c_{1}(z)=c_{1}\left(z_{0}\right)+\int_{\left.u_{u}\right)}^{u} d u^{\prime} \gamma^{\prime}\left(u^{\prime}\right) a_{12}\left(\gamma\left(u^{\prime}\right)\right) e^{i\left(1 \Delta _ { 1 2 } \left(\gamma^{\prime}\left(u^{\prime}\right)\right.\right.} c_{2}\left(\gamma\left(u^{\prime}\right)\right)
$$

and a similar expression holds for (2.31). From these equations and (2.28), we obtain

$$
\lim _{t \rightarrow \pm x_{-}} c_{k}(t+i s)=c_{k}( \pm \infty), \quad k=1,2,|s|<a
$$

with $c_{k}( \pm \infty)$ independent of $s$.

\subsection{Analytic Continuation of $U(z)$}

The set $M=S_{a} \backslash X$, where $X$ is the set of eigenvalue-crossings, is not simply connected. The eigenvalues $e_{k}$ and eigenprojections $P_{k}$ have analytic extensions on $M$, but these analytic extensions are multi-valued. However, the operator $K(z)$ defined in $(2.9)$ is a single-valued analytic operator on $M$. At the points of $X, K(z)$ has simple poles. Thus $K(z)$ is meromorphic on $S_{a}$ and the equation

$$
U^{\prime}(z)=K(z) U(z)
$$

is well defined, and has regular singular points at the eigenvalue-crossings. The solutions of (2.34) are in general multi-valued.

These solutions allow us to define new families of $z$-dependent basis. We are interested in the solution of (2.34) which is the analytic extension of the solution $U(z)$ defined on $\Omega$ in the previous section. This solution is determined by the initial value at $z=0, U(0)=\mathbb{1}$. Let $\gamma_{1}$ and $\gamma_{2}$ be two closed paths in $M$ based at 0 . We do the analytic continuation of $U$ along the paths $\gamma_{1}$ and $\gamma_{2}$. Coming back at 0 the values of these analytic continuations are $U\left(0 \mid \gamma_{1}\right)$ and $U\left(0 \mid \gamma_{2}\right)$. They depend only on the homotopy class of $\gamma_{1}$ and $\gamma_{2}$. If $\gamma_{2} \cdot \gamma_{1}$ represents the closed path at 0 by first going $\gamma_{1}$ and then $\gamma_{2}$, we have

$$
U\left(0 \mid \gamma_{2} \cdot \gamma_{1}\right)=U\left(0 \mid \gamma_{2}\right) U\left(0 \mid \gamma_{1}\right)
$$

Thus $U(0 \mid \gamma)$ gives a representation of the fundamental group $\Pi_{1}(M ; 0)$ of $M$ at 0 . Note that if $\gamma$ is parametrized by $u \in[0,1]$, then

$$
U(0 \mid \gamma)=\llbracket+\sum_{n \geqslant 1} \int_{0}^{1} d u_{n} \int_{0}^{u_{n}} d u_{n-1} \cdots \int_{0}^{u_{2}} d u_{1} K\left(\gamma\left(u_{n}\right)\right) \dot{\gamma}\left(u_{n}\right) \cdots K\left(\gamma\left(u_{1}\right)\right) \dot{\gamma}\left(u_{1}\right) .
$$


Let now $\gamma_{1}$ and $\gamma_{2}$ be two paths starting at $z=0$ and ending at $z$. The values of the analytic continuations of $U$ along $\gamma_{1}\left(\operatorname{resp} . \gamma_{2}\right)$ at the point $z$ are $U\left(z \mid \gamma_{1}\right)$ (resp. $\left.U\left(z \mid \gamma_{2}\right)\right)$. They are related as

$$
\begin{aligned}
U\left(z \mid \gamma_{2}\right) & =U\left(z \mid \gamma_{1}\right) U\left(z \mid \gamma_{1}\right){ }^{1} U\left(z \mid \gamma_{2}\right) \\
& =U\left(z \mid \gamma_{1}\right) U\left(z \mid \gamma_{1}{ }^{1} \cdot \gamma_{2}\right) .
\end{aligned}
$$

where $\gamma_{1}^{-1} \cdot \gamma_{2}$ is a closed path at 0 . Let $\left\{\varphi_{1}(0), \varphi_{2}(0)\right\}$ be the basis of orthonormal eigenvectors of $H(0)$ which we have chosen in the preceding section. Let $\gamma$ be a simple closed loop based at 0 around the branching point $z_{1}$. We can transport the basis $\left\{\varphi_{1}(0), \varphi_{2}(0)\right\}$ along $\gamma$. Coming back to the base point we have a new basis

$$
\left\{U(0 \mid \gamma) \varphi_{1}(0), U(0 \mid \gamma) \varphi_{2}(0)\right\} .
$$

Both basis vectors in (2.38) are eigenvectors of $H(0)$. Since $\gamma$ is a simple closed loop,

$$
H(0)\left(U(0 \mid \gamma) \varphi_{1}(0)\right)=e_{2}(0)\left(U(0 \mid \gamma) \varphi_{1}(0)\right)
$$

and

$$
H(0)\left(U(0 \mid \gamma) \varphi_{2}(0)\right)=e_{1}(0)\left(U(0 \mid \gamma) \varphi_{2}(0)\right) .
$$

We define two phases $\theta_{k i}(0 \mid \gamma), k \neq j$, by

$$
U\left(0 \mid \gamma^{\prime}\right) \varphi,(0)=e^{-i H_{k}\left(0 \mid \gamma^{\prime}\right)} \varphi_{k}(0) \text {. }
$$

In general the phases are complex and therefore the new basis vectors are not normalized. An explicit formula $\theta_{k j}(0 \mid \gamma)$ in terms of the functions $B_{1}, B_{2}$, and $B_{3}$ is given in appendix. Let us denote by

$$
\varphi_{k}(z \mid \gamma):=U(z \mid \gamma) \varphi_{k}(0)
$$

the vector which one obtains by transporting $\varphi_{k}(0)$ along $\gamma$, where $\gamma$ is a path from 0 to $z$. Using (2.41) and (2.37) we have the following relation if $\gamma_{1}$ and $\gamma_{2}$ are two

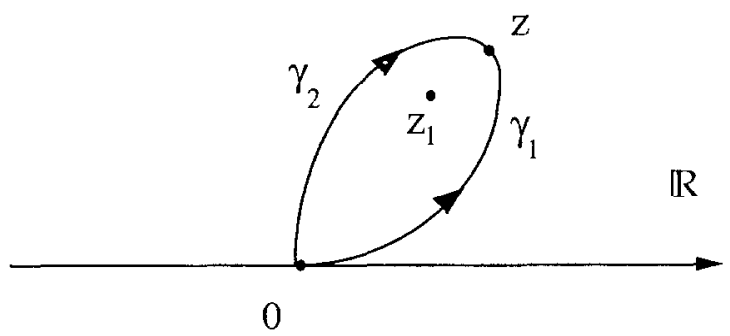

Fig. 3. The paths $\gamma_{1}$ and $\gamma_{2}$. 
paths (Fig. 3) from 0 to $z$ and such that $\gamma_{1}^{-1} \cdot \gamma_{2}$ is a simple closed path at 0 around $z_{1}$ :

$$
\varphi_{k}\left(z \mid \gamma_{2}\right)=e^{-i \theta_{k k}\left(0 \mid \gamma_{1}^{-1} \gamma_{2}\right)} \varphi_{j}\left(z \mid \gamma_{1}\right), \quad k \neq j .
$$

\subsection{Transition Probability in the Adiabatic Limit}

In this section we determine the coefficient $c_{2}(+\infty)=\lim _{t \rightarrow x} c_{2}(t)$ of the solution $\psi_{T}$ of Eq. (2.5) subject to the boundary condition (2.6). This allows us to compute the transition probability

$$
\lim _{t \rightarrow \infty}\left\|P_{2}(t) \psi_{T}(t)\right\|^{2}=\left|c_{2}(+\infty)\right|^{2}
$$

and to prove the formula (1.6).

The method consists in controlling the solution $\psi_{T}$ along a path $t \mapsto \tilde{\gamma}(t) \in S_{a}$ parametrized by $t \in \mathbb{R}$ which has the following properties:

(i) $\lim _{t \rightarrow \pm x} \operatorname{Re} \tilde{\gamma}(t)= \pm \infty, \lim _{t \rightarrow \pm \infty} \operatorname{Im} \tilde{\gamma}(t)=s_{ \pm},\left|s_{ \pm}\right|<a$

(ii) $\tilde{\gamma}$ goes over the branching point $z_{1}$ in the upper half plane.

Since $\tilde{\gamma}$ is not in $\Omega$ we cannot use the decomposition (2.24)

$$
\psi_{T}(z)=\sum_{j=1}^{2} c_{j}(z) e^{-i T \lambda_{j}(z)} \varphi_{j}(z)
$$

along all the path $\tilde{\gamma}$. But we can use it for $t \ll-1$. Then we make an analytic continuation of (2.45) along $\tilde{\gamma}$. The resulting decomposition is written

$$
\psi_{T}(z)=\sum_{j=1}^{2} \tilde{c}_{j}(z) e^{-i T \tilde{\lambda_{j}}(z)} \tilde{\varphi}_{j}(z)
$$

where $\tilde{f}$ means that we have made an analytic continuation of $f$ along $\tilde{\gamma}$. The coefficients $\widetilde{c_{k}}(z)$ now satisfy the analytic continuation of Eqs. (2.30) and (2.31) along $\tilde{\gamma}$. Let $z$ be some point of $\tilde{\gamma}$ with $t \gg 1$ (see Fig. 4). Using (2.43) of Section 2.2, we see that

$$
\tilde{\varphi}_{1}(z)=e^{\cdot i \theta_{21}(0 \mid \eta)} \varphi_{2}(z)
$$

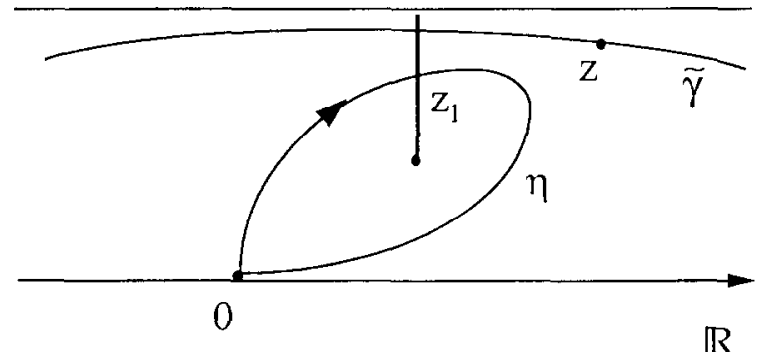

FIG. 4. The paths $\tilde{i}, \eta$ and the point $z \in \tilde{i}$. 
and

$$
\widetilde{\varphi}_{2}(z)=e^{-i \theta_{12}(0 \mid \eta)} \varphi_{1}(z),
$$

where $\eta$ is the simple closed path at 0 of Fig. 4, which is homotopic to the path $\gamma_{1}{ }^{-1} \gamma_{2}$ of (2.43). Comparing (2.45) and (2.46), we obtain

$$
\begin{aligned}
& \psi_{T}(z)=c_{1}(z) e^{-i T \lambda_{1}(z)} \varphi_{1}(z)+c_{2}(z) e^{\left.-i T \lambda_{3} l z\right)} \varphi_{2}(z)
\end{aligned}
$$

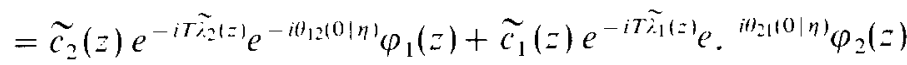

and therefore we have the relation

$$
\begin{aligned}
c_{2}(z) & =e^{-i \theta_{21}(0 \mid \eta)} e^{-i T \tilde{\tilde{\lambda}_{1}}(z)+i T \hat{\lambda}_{2}(z)} \widetilde{c_{1}}(z) \\
& =\exp \left(-i \theta_{21}(0 \mid \eta)\right) \exp \left(-i T \int_{\eta} e_{1}\right) \widetilde{c_{1}}(z),
\end{aligned}
$$

where $\int_{\eta} e_{1}$ is the integral over $\eta$ of the analytic continuation of $e_{1}$ along $\eta$. Similarly, we have

$$
c_{1}(z)=\exp \left(-i \theta_{12}(0 \mid \eta)\right) \exp \left(-i T \int_{\eta} e_{2}\right) \tilde{c}_{2}(z) .
$$

If we can control $\widetilde{c_{1}}$ then we obtain information on $c_{2}$.

The equation for $\widetilde{c_{1}}$ is given in (2.32), where we must replace $\gamma$ by $\tilde{\gamma}$ and all quantities appearing in (2.32) are defined by analytic continuation along $\tilde{\gamma}$. We introduce the notations $\widetilde{c_{k}}(t)$ for $\widetilde{c_{k}}(\tilde{\gamma}(t)), \widetilde{\Delta}_{i j}(t)$ for ${\widetilde{u_{i j}}}_{(\tilde{\gamma}}(t))$, and $\widetilde{a}_{i j}(t)$ for $\widetilde{a}_{i j}(\tilde{\gamma}(t))$. The boundary condition (2.6) is equivalent to $\lim _{t \rightarrow-\infty} \widetilde{c}_{1}(t)=c_{1}(-\infty),\left|c_{1}(-\infty)\right|=1$, and $\lim _{t \rightarrow-s_{2}} \widetilde{c_{2}}(t)=0$. With these notations the equations for $\widetilde{c_{1}}$ and $\widetilde{c_{2}}$ read

$$
\widetilde{c_{1}}(t)=c_{1}(-\infty)+\int_{x}^{t} d u \dot{\gamma}(u) \widetilde{a_{12}}(u) e^{i T \widetilde{d_{12}}(u)} \widetilde{c_{2}}(u)
$$

and

$$
\widetilde{c_{2}}(t)=\int_{-}^{l} d u \dot{\gamma}(u) \tilde{a_{21}}(u) e^{i T \cdot \tilde{u_{21}}(u)} \tilde{c_{1}}(u) .
$$

We perform an integration by parts in Eq. (2.52) in order to use the fact that $T$ is a large parameter:

$$
\begin{aligned}
\tilde{c_{1}}(t)= & c_{1}(-\infty)+\frac{1}{i T} \frac{\widetilde{a_{12}}(t)}{\widetilde{\Delta_{12}^{\prime}}(t)} e^{i T \tilde{s_{12}(t)} \tilde{c_{2}}(t)} \\
& -\frac{1}{i T} \int_{\infty}^{t} d u \dot{\tilde{\gamma}}(u)\left(\frac{\widetilde{a_{12}}}{\widetilde{\Delta_{12}}}\right)^{\prime}(u) e^{i T \tilde{d_{12}}(u)} \tilde{c_{2}}(u) \\
& -\frac{1}{i T} \int_{-\infty}^{t} d u \dot{\tilde{\gamma}}(u) \frac{\widetilde{a_{12}} \widetilde{a_{21}}}{\widetilde{\Delta_{12}^{\prime}}}(u) \widetilde{c_{1}}(u) .
\end{aligned}
$$


We recall that' means $d / d z$ so that

$$
\widetilde{\Delta_{1}^{\prime},}(u)=\left.\frac{d}{d z} \widetilde{\Delta_{1},}(z)\right|_{z=\tilde{\gamma}(u)} .
$$

Up to this point, we have only used the property that $\tilde{\gamma}$ must go over the branching point $z_{1}$, in order to get the essential relation $(2.50)$. To treat the second term in (2.54), we suppose that $\tilde{\gamma}$ satisfies the new condition VI.

VI. Dissipative path. The path $\tilde{\gamma}$ such that $\operatorname{Im} \widetilde{\Delta_{12}}(u)$ is always a nondecreasing function of $u$.

Condition VI is a strong condition since it is a global condition on $\tilde{\gamma}$. Such a condition is typical in the WKB method. Using it, we can estimate $\tilde{c}_{2}$. Indeed, from (2.53), we have

$$
\begin{aligned}
& \left|\widetilde{c_{2}}(t)\right| \leqslant \int_{-x}^{t} d u|\dot{\tilde{\gamma}}(u)|\left|\widetilde{a_{21}}(u)\right| e^{-T \operatorname{lm} \tilde{d_{21}(u)}}\left|\widetilde{c_{1}}(u)\right| \\
& \leqslant e^{-T \operatorname{Im} \widetilde{d_{21}(t)}}\left\|\widetilde{c_{1}}\right\|_{\infty_{1}}\left\|\tilde{a_{21}}\right\|_{1}\|\dot{\hat{\gamma}}\|_{\infty} \text {. }
\end{aligned}
$$

Condition II implies that $\left\|\widetilde{a_{21}}\right\|_{1}<\infty$ (the proof is the same as for (2.28)). Similarly, $\left\|\widetilde{a_{21}} / \widetilde{\Delta_{12}^{\prime}}\right\|_{1}<\infty$. Since $\widetilde{a_{21}} / \widetilde{\Delta_{12}^{\prime}}$ is an analytic function of $z$, we also conclude by the Cauchy formula that $\left\|\left(\tilde{a_{21}} / \widetilde{A_{12}^{\prime}}\right)^{\prime}\right\|_{1}<\infty$. From $(2.54)$ and (2.56) we have $\left\|\tilde{c}_{1}\right\|_{\infty}(1-c / T) \leqslant 1$, provided $T$ is large enough, with $c$ some constant. Thus

$$
\sup _{t}\left|\tilde{c}_{1}(t)-c_{1}(-\infty)\right| \leqslant C / T
$$

for another constant $C$. From (2.57) and (2.50), we obtain the desired result (1.6),

$$
\begin{aligned}
\lim _{t \rightarrow+\infty}\left\|P_{2}(t) \psi_{T}(t)\right\|^{2} & =\lim _{t \rightarrow+\infty}\left|c_{2}(t)\right|^{2} \\
& =\exp \left(2 \operatorname{Im} \theta_{21}(0 \mid \eta)\right) \exp \left(2 T \operatorname{Im} \int_{\eta} e_{1}\right)(1+O(1 / T))
\end{aligned}
$$

Equation (2.58) is the basic result of this section.

Remarks. (i) We have used a path $\tilde{y}$ going over $z_{1}$ because the energy of the system at $t=-\infty$ is $e_{1}(-\infty)$ which is the lowest energy level. If we had taken at $t=-\infty$ the solution $\psi_{T}$ such that $\lim _{t \rightarrow-\infty}\left\|P_{2}(t) \psi_{T}(t)\right\|=1$ then we should do the same analysis with a path $\tilde{\gamma}$ going below the branching point $\overline{z_{1}}$. The result corresponding to $(1.6)$ in this case reads

$$
\lim _{t \rightarrow+\infty}\left\|P_{1}(t) \psi_{T}(t)\right\|^{2}=\exp \left(2 \operatorname{Im} \theta_{12}(0 \mid \bar{\eta})\right) \exp \left(2 T \operatorname{Im} \int_{\bar{\eta}} e_{2}\right)(1+O(1 / T))
$$


where $\bar{\eta}$ is the simple closed path based at 0 which is obtained from $\eta$ by complex conjugation. We have $\operatorname{Im} \int_{\eta} e_{1}=\operatorname{Im} \int_{\bar{\eta}} e_{2} \leqslant 0$ and $\operatorname{Im} \theta_{12}(0 \mid \bar{\eta})=\operatorname{Im} \theta_{21}(0 \mid \eta)$.

(ii) In formula (1.6) we have chosen some point $x_{0} \in \mathbb{R}$. Of course, this choice of $x_{0}$ has no influence on the final result. If $\eta_{1}$ is a closed path based at 0 around $z_{1}$ and $\eta_{2}$ is a closed path based at $x_{0}$ around $z_{1}$, which is obtained by a continuous deformation of $\eta_{1}$, then

$$
U\left(x_{0} \mid \eta_{2}\right)=M U\left(0 \mid \eta_{1}\right) M^{*}
$$

where $M$ is a unitary matrix. Consequently,

$$
\operatorname{Im} \theta_{k j}\left(x_{0} \mid \eta_{2}\right)=\operatorname{Im} \theta_{k j}\left(0 \mid \eta_{1}\right)
$$

\subsection{Geometry and Transition Probability}

In the last section we have proven the generalized Dykhne formula (2.58) for the transition probability under conditions I, II, II, V, and VI. The first three conditions describe the framework in which the problem is studied. Condition $\mathrm{V}$ is now replaced by condition $\mathrm{V}^{\prime}$.

$\mathrm{V}^{\prime}$. Eigenvalue-crossings (generic case). The set $X^{\prime}$ of zeros of $\rho(z)$ in $S_{a}$ consists of $n$ pairs of points $z_{k}$ and $\overline{z_{k}}, k=1, \ldots, n$. All zeros are simple. By convention $\operatorname{Im} z_{k}>0, k=1, \ldots, n$.

Finally, condition VI appears as a technical but essential condition for the proof of (2.58). In this section we analyze this condition from a geometric point of view. This analysis allows us to express the formula giving the transition probability in purely geometric terms. We close this section by discussing concrete examples.

The function $\rho(z)=B_{1}^{2}(z)+B_{2}^{2}(z)+B_{3}^{2}(z)$ is holomorphic in the simply connected domain $S_{a}$. By condition $\mathrm{V}^{\prime}, \rho(z)$ has $2 n$ simple zeros in $S_{u}$. This holomorphic function defines a quadratic differential $\rho(z) d^{2} z$ on $S_{a}$. The behaviour of the trajectories of the quadratic differential as well as the metric associated with it play an important role in our analysis of the transition probability. We recall the definition of these concepts and their main properties in our present situation. We follow closely Strebel's monograph [13].

A point $z \in S$ is called regular if $\rho(z) \neq 0$ and it is called critical if $\rho(z)=0$. A $\theta$-straight arc is a smooth curve $t \mapsto \gamma(t)$ in $S_{a}$ such that for all $t$,

$$
\arg \left(\rho(\gamma(t)) \dot{\gamma}^{2}(t)\right)=\theta=\text { const. }
$$

Notice that this implies in particular that $\rho(\gamma(t)) \neq 0$ and therefore a straight arc contains only regular points. If $\theta=0$ the straight arc is horizontal and if $\theta=\pi$ it is vertical. A maximal horizontal are is called a trajectory of the quadratic differential $\rho$. On a trajectory we have

$$
\rho(\gamma(t)) \dot{\gamma}^{2}(t)>0
$$


and by a reparametrization of the trajectory we see that it is a maximal solution of the differential equation

$$
\rho(z)\left(\frac{d z}{d u}\right)^{2}=1
$$

In a small disc $V$ around 0 we can define a single-valued function $\Phi(z)$ by choosing a branch of the square root and by setting

$$
\Phi(z)=\int_{0}^{z} \sqrt{\rho\left(z^{\prime}\right)} d z^{\prime} .
$$

Since the eigenvalues of the hamiltonian $H(z)$ are $\pm \frac{1}{2} \sqrt{\rho(z)}$ we have $\sqrt{\rho\left(z^{\prime}\right)}=$ $\pm\left(e_{1}\left(z^{\prime}\right)-e_{2}\left(z^{\prime}\right)\right)$. We choose the definition

$$
\Phi(z)=\int_{0}^{z}\left(e_{1}\left(z^{\prime}\right)-e_{2}\left(z^{\prime}\right)\right) d z^{\prime} .
$$

In (2.65) and (2.66), the integration path is in $V$. We also denote by $\Phi$ the multivalued function on $S_{a} \backslash X$ obtained by analytic continuation. Let $z_{0} \in S_{a} \backslash X$. A $\rho$-disc with center $z_{0}$ and radius $r$ is a region $U \subset S_{a} \backslash X$ containing $z_{0}$ which is mapped homeomorphically onto a disc of radius $r$ by a branch of $\Phi, z_{0}$ being mapped on the center of the disc. The value at $z$ of a branch of $\Phi$ on a $\rho$-disc $U$ with center $z_{0}$ is given by

$$
w_{0}+\int_{z_{0}}^{z}\left(e_{1}\left(z^{\prime}\right)-e_{2}\left(z^{\prime}\right)\right) d z^{\prime}
$$

or by

$$
w_{0}-\int_{-0}^{z}\left(e_{1}\left(z^{\prime}\right)-e_{2}\left(z^{\prime}\right)\right) d z^{\prime},
$$

where in (2.67) and (2.68) $w_{0}$ is the value of the branch at $z_{0}$ and the integration path is in $U$. In the $\rho$-disc $U$ we can solve the differential equation (2.64) with initial condition $z^{*} \in U$ at $u_{0}$. The solution $u \mapsto \gamma(u)$ satisfies the equations

$$
\left(u-u_{0}\right)= \pm \int_{z^{*}}^{r u)}\left(e_{1}\left(z^{\prime}\right)-e_{2}\left(z^{\prime}\right)\right) d z^{\prime} .
$$

If we still denote by $\Phi(z)$ a branch of the function $\Phi$ on $U$ we have

$$
\left(u-u_{0}\right)+\Phi\left(z^{*}\right)=\Phi(\gamma(u))
$$

and the solution of (2.64), which is the horizontal straight arc passing through $z^{*}$, is the level-line in $U$ of $\operatorname{Im} \Phi$,

$$
\operatorname{Im} \Phi(\gamma(u))=\operatorname{Im} \Phi\left(z^{*}\right) .
$$


Therefore, in a $\rho$-disc $U$ of radius $r$ around $z_{0}$ the set of trajectories is homeomorphic to a set of horizontal lines in a disc of radius $r$. In particular, two different trajectories cannot cross. We can parametrize globally the trajectory $\alpha$ passing through $z_{0}$ as follows. We choose a parametrization $u \mapsto \alpha(u)$ such that $\alpha(0)=z_{0}$. From $(2.70)$ we get locally, i.e., for $|u|<r$,

$$
x(u)=\Phi^{-1}\left(w_{0}+u\right) .
$$

Then we analytically continue the branch of $\Phi$ along $\alpha$. We obtain a function $\Phi_{\alpha}$ whose restriction on $\alpha$ itself is injective, as a consequence of (2.63). The image of $\alpha$ by $\Phi_{x}$ is the set

$$
\left\{w=w_{0}+u ; u \in\left(u_{-}, u_{+}\right) \subset \mathbb{R}\right\},
$$

where the interval $\left(u_{-}, u_{+}\right)$is maximal and we have for all $u \in\left(u, u_{+}\right), \alpha(u)=$ $\Phi_{\alpha}{ }^{1}\left(w_{0}+u\right)$. This parametrization is called a natural parametrization of $\alpha$. By definition a trajectory is called critical if $\lim _{u \rightarrow u_{-}} \alpha(u)$ or $\lim _{u \rightarrow u_{+}} \alpha(u)$ is a critical point of $\rho$. In a neighbourhood of a critical point $z_{1}$ the set of trajectories of $\rho$ is homeomorphic to the set of level-lines of $\operatorname{Im} z^{3 / 2}$ in a neighbourhood of $z=0$. In particular there are three horizontal straight arcs which have $z_{1}$ as accumulation point (see Fig. 5).

Remark. The critical trajectories are called sometimes Stokes lines (see [14]). These lines play an essential role in the analysis of the WKB method.

We can also introduce a metric associated with the quadratic differential $\rho$. Let $\gamma$ be some rectifiable curve. We define the $\rho$-length of $\gamma$ by

$$
||_{1}=\int_{;}|\rho(z)|^{1 / 2}|d z|
$$

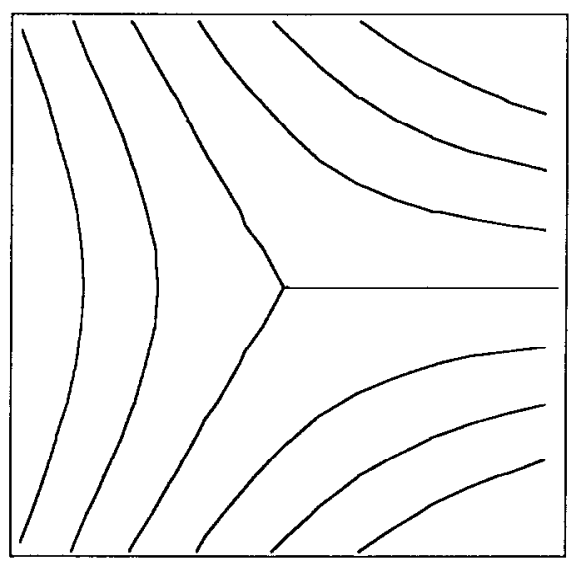

FIG. 5. Level-lines of $\operatorname{Im} z^{3} 2$ around $z=0$. 
If $z_{1}$ and $z_{2} \in S_{a}$ then the $\rho$-distance $d_{\rho}\left(z_{1}, z_{2}\right)$ of $z_{1}$ and $z_{2}$ is given by the infimum of $|\gamma|_{\rho}$, where $\gamma$ is a rectifiable curve from $z_{1}$ to $z_{2}$ in $S_{a}$. If $\gamma$ is contained in a $\rho$-disc $U$ of radius $r$ then its $\rho$-length $|\gamma|_{\rho}$ is equal to the Euclidean length of the image of $\gamma$ by a branch of $\Phi$ defined on $U$. In particular, a $\rho$-disc of radius $r$ is a disc of radius $r$ in the $\rho$-metric. A natural parametrization of $\alpha$ is essentially the parametrization of the arc-length for the $\rho$-metric. Finally, we call a curve $t \mapsto \gamma(t)$, $a<t<b$, a geodesic if it is locally shortest. This means that for every $t$ there is an interval $\left[t_{1}, t_{2}\right]$ such that $t \in\left[t_{1}, t_{2}\right]$ and the arc $\gamma\left(\left[t_{1}, t_{2}\right]\right)$ is the shortest connection of the two points $\gamma\left(t_{1}\right)$ and $\gamma\left(t_{2}\right)$. In a neighbourhood of any regular point a geodesic is a $\theta$-straight arc.

The main tool for analyzing the set of the critical trajectories is Teichmueller's lemma. A geodesic polygon is a curve $\gamma$ composed of open straight arcs and their end points which can be critical points of $\rho$. We quote Teichmueller's lemma from [15]. A more general version for meromorphic quadratic differential is given in $[13]$.

LEMMA 2.1 (Teichmueller's lemma). Let $\rho$ be holomorphic in the closure of a domain $A$ in the complex plane which is bounded by a simple closed geodesic polygon in the $\rho$-metric, whose sides $\gamma_{j}$ form interior angles $\theta_{j}$ at the vertices, $0<\theta_{j} \leqslant 2 \pi$. If $m_{i}$ and $n_{j}$ denote the orders of the zeros of $\rho$ in $A$ and on $\partial A$, respectively, then

$$
\sum_{j}\left(1-\left(n_{j}+2\right) \frac{\theta_{j}}{2 \pi}\right)=2+\sum_{i} m_{i} .
$$

This lemma is a consequence of the argument principle.

Let $\tilde{\gamma}$ be a dissipative path in the upper half-plane so that the generalized Dykhne formula (2.58) can be proven using $\tilde{\gamma}$ as in Section 2.3. We have

THEOREM 2.1. Let $\tilde{\gamma}$ be as above. Then

(1) $\tilde{\gamma}$ is a simple curve.

(2) The open region $\Sigma$ in $S_{a}$ between the path $\tilde{\gamma}$ and the real axis contains exactly one eigenvalue-crossing, say $z_{1}$.

(3) There are three distinct critical trajectories $\alpha_{1}, \alpha_{2}, \alpha_{3}$ having $z_{1}$ as accumulation point.

(4) The trajectory $\alpha_{1}$ is entirely inside $\sum$ and has a natural parametrization with $u \in(-\infty, 0)$ so that $\lim _{u \rightarrow 0} \alpha_{1}(u)=z_{1}, \lim _{u \rightarrow} \quad$ $\operatorname{Re} \alpha_{1}(u)=-\infty$.

(5) The trajectory $\alpha_{2}$ is entirely inside $\Sigma$ and has a natural parametrization with $u \in(0, \infty)$ so that $\lim _{u \rightarrow 0} \alpha_{2}(u)=z_{1}, \lim _{u \rightarrow x_{0}} \operatorname{Re} \alpha_{2}(u)=\infty$.

(6) The trajectory' $\alpha_{3}$ has a natural parametrization with $u \in\left(0, u_{+}\right)$so that $\lim _{u \rightarrow 0} \alpha_{3}(u)=z_{1}$. The set of $u \in\left(0, u_{+}\right)$such that $\alpha_{3}(u)$ is a point of $\tilde{\gamma}$ is a non-empty connected set.

Remark. If we define a curve $u \mapsto g(u)$ by $g(u)=\alpha_{1}(u)$ when $u<0, g(u)=\alpha_{2}(u)$ 
when $u>0$ and $g(0)=z_{1}$, then $g$ is an horizontal geodesic inside $\Sigma$ passing through the critical point $z_{1}$. The main statement of Theorem 2.1 is the existence of only one critical point $z_{1}$ in $\Sigma$ and of the horizontal geodesic through $z_{1}$. Let $\Sigma_{1}$ be the region in $S_{a}$ defined by

$$
\Sigma_{1}=\{z=u+i v|| v \mid<\operatorname{Im} g(u), u \in \mathbb{R}\} .
$$

By the argument principle one can see that the analytic continuation of the function $\Phi(z)$ on $\Sigma_{1}$ is univalent. The image of $\Sigma_{1}$ by $\Phi$ is an horizontal open strip of width $2\left|\operatorname{Im} \Phi\left(z_{1}\right)\right|$, where $\Phi\left(z_{1}\right)$ is defined by continuity. In particular the $\rho$-distance to the real axis is

$$
d_{\rho}\left(z_{1}, \mathbb{R}\right)=\left|\operatorname{Im} \Phi\left(z_{1}\right)\right|=\left|\operatorname{Im} \Delta_{12}\left(z_{1}\right)\right|
$$

since $\Phi(z)$ coincide with the function $\Delta_{12}(z)$ defined in Scction 2.3. Morcover, for any other $z_{k}, k \neq 1$,

$$
d_{\rho}\left(z_{k}, \mathbb{R}\right)>d_{\rho}\left(\bar{z}_{1}, \mathbb{R}\right) .
$$

Note that formula (2.76) holds because on $\Sigma_{1}, \Phi$ is univalent. In general $\Phi$ is multivalued and we cannot expect such a formula for $d_{\rho}\left(z_{k}, \mathbb{R}\right), k \neq 1$.

Proof. (1) We assume that $\tilde{\gamma}$ is parametrized by $t \in \mathbb{R}$. Let $\tilde{\Delta_{12}}$ be the branch of $\Phi$ such that $\operatorname{Im} \widetilde{\Delta_{12}}(\tilde{\gamma}(t))$ is non-decreasing in $t$ (see Section 2.3). We choose $t_{1}$ and $t_{2}>t_{1}$ and consider only the part of $\tilde{\gamma}$ for $t \in\left[t_{1}, t_{2}\right]$. The image of this part of $\tilde{\gamma}$ by $\widetilde{\Delta_{12}}$ is a simple path from $w_{1}=\widetilde{A_{12}}\left(\tilde{\gamma}\left(t_{1}\right)\right)$ to $w_{2}=\widetilde{\Delta_{12}}\left(\tilde{\gamma}\left(t_{2}\right)\right)$. Indeed, if $\operatorname{Im} \widetilde{\Delta_{12}}(\tilde{\gamma}(t))$ is constant on some interval then $\operatorname{Re} \widetilde{\Delta_{12}}(\tilde{\gamma}(t))$ is strictly increasing or decreasing on that interval. Elsewhere, $\operatorname{Im} \widetilde{\Delta_{12}}(\tilde{\gamma}(t))$ is increasing. We can approximate this path from $w_{1}$ to $w_{2}$ by a polygonal line $t \mapsto p(t)$ made of horizontal and vertical Euclidean segments such that $\operatorname{Im} p(t)$ is nondecreasing. Taking the image by ${\widetilde{\Delta_{12}}}^{-1}$ of this line we get a geodesic polygonal line in the $\rho$-geometry which approximates the path $\tilde{\gamma}$ for $t \in\left[t_{1}, t_{2}\right]$ (the inverse map $\widetilde{\Delta_{12}}{ }^{1}$ is well defined locally).

Let us suppose that $\tilde{\gamma}$ is not simple. Then there exist $t_{1}$ and $t_{2}>t_{1}$ so that $\tilde{\gamma}\left(t_{1}\right)=\tilde{\gamma}\left(t_{2}\right)$. We suppose that $t \in\left[t_{1}, t_{2}\right] \mapsto \tilde{\gamma}(t)$ is a simple closed path. This path can be approximated by a simple closed geodesic polygon. The interior angles of this polygon are equal to $\pi / 2$ or $3 \pi / 2$. Since $\operatorname{Im} p(t)$ is nondecreasing the number of interior angles with $\theta_{i}=\pi / 2, N(\pi / 2)$, and the number of interior angles $\theta_{j}=3 \pi / 2$, $N(3 \pi / 2)$, satisfy the inequality

$$
|N(3 \pi / 2)-N(\pi / 2)| \leqslant 2 .
$$

Applying Teichmueller's lemma we get a contradiction. This proves (1).

(2) The geometry of the trajectories is well understood in the regions $|\operatorname{Im} z|<a$ and $|\operatorname{Re} z|$ large enough. Indeed, in these regions $\rho$ is essentially constant and tends to positive values as $|\operatorname{Re} z| \rightarrow \infty$. Therefore, the $\rho$-geometry is essentially 
the Euclidean geometry. The trajectories are essentially horizontal in the Euclidean sense and the vertical straight arcs are essentially vertical lines in the Euclidean sense. Thus we can find a pair of points $x_{1}$, on the negative real axis, and $z_{1}=\tilde{\gamma}\left(t_{1}\right)$ which can be joined by a vertical straight arc $\gamma_{1}$ in $\Sigma$. Similarly there exists a pair of points $x_{2}$, on the positive real axis, and $z_{2}=\tilde{\gamma}\left(t_{2}\right)$ which can be joined by a vertical straight arc $\gamma_{2}$ in $\Sigma$. Moreover, the curve $\Gamma$ which is composed of $i_{1}, \gamma_{2}$, $\left[x_{1}, x_{2}\right]$, and the part of $\tilde{\gamma}$ for $t \in\left[t_{1}, t_{2}\right]$ can be assumed to be simple and closed. For later purposes we denote by $\Sigma^{\prime}$ the bounded region with boundary $\Gamma$. As above we approximate the curve by a geodesic simple closed polygon. In this case we have

$$
|N(3 \pi / 2)-N(\pi / 2)| \leqslant 6
$$

and by Teichmueller's lemma there is at most one critical point in $\Sigma$. There is in fact exactly one critical point, otherwise (2.47) and (2.48) could not be true.

(3) If $\alpha_{1}$ and $\alpha_{2}$ are not distinct, then they coincide. Let us suppose that this is the case. Then $\alpha_{1}$ and $z_{1}$, the critical point in $\Sigma$, form a simple closed geodesic polygon. Applying Teichmueller's lemma we get a contradiction.

(4) Let us suppose that $\tilde{\gamma}$ intersects $\alpha_{1}$ at $\tilde{\gamma}\left(t_{1}\right)$ and $\alpha_{2}$ at $\tilde{\gamma}\left(t_{2}\right)$. We may suppose that $t_{1}<t_{2}$. Let $\alpha^{\prime}$ be the part of $\alpha_{1}$ between $z_{1}$ and $\tilde{\gamma}\left(t_{1}\right)$ and $\alpha^{\prime \prime}$ be the part of $\alpha_{2}$ between $\tilde{\gamma}\left(t_{2}\right)$ and $z_{1}$. Then the path $\alpha^{\prime}$ followed by the part of $\tilde{\gamma}$ with $t \in\left[t_{1}, t_{2}\right]$ and then followed by $\alpha^{\prime \prime}$ is a path along which $\operatorname{Im} \Phi$ is nondecreasing for some branch of $\Phi$ (we choose the branch of $\Phi$ which is defined by analytic continuation along this path and which coincides with $\tilde{\Delta_{12}}$ on $\tilde{\gamma}$ when $\left.t \in\left[t_{1}, t_{2}\right]\right)$. By point (1) this path is simple and if we add $z_{1}$ to it we obtain a simple closed path which can be approximated by a simple closed geodesic polygon. The angle at $z_{1}$, the critical point, is $2 \pi / 3$ and $(2.78)$ holds for the other angles. Thus we obtain a contradiction from Teichmueller's lemma. Notice that the same argument shows that the set of intersection points of any $\alpha_{i}$ with $\tilde{\gamma}$ is connected.

Let us consider the set $\Sigma^{\prime}$ with boundary $\Gamma$ defined in (2). Let $A(z)$ be the analytic continuation in $\Sigma^{\prime} \backslash\left\{z_{1}\right\}$ of the function

$$
2 \int_{z_{1}} e_{1}\left(z^{\prime}\right) d z^{\prime}
$$

defined in a neighbourhood $U$ of $z_{1}$ (the integration path being in $U$ ). This function is 2-valued since $z_{1}$ is the only critical point and there exists $C<\infty$ such that

$$
|\operatorname{Re} A(z)| \leqslant C
$$

for any $z \in \Sigma^{\prime} \backslash\left\{z_{1}\right\}$ and any value $\Delta(z)$ over $z$. If we follow a branch of $\Delta(z)$ along $\alpha_{i}$ then $\operatorname{Re} A$ is strictly increasing or strictly decreasing. Inequality (2.81) implies that $\alpha_{i}$ must intersect $\Gamma$. However, each vertical straight arc $\gamma_{i}$ of $\Gamma$ can be intersected by at most one $\alpha_{i}$ and only once. (This follows again from Teichmueller's lemma.) Since the real interval $\left[x_{1}, x_{2}\right]$ is a horizontal straight are it cannot be intersected by $\alpha_{1}, \alpha_{2}$, or $\alpha_{3}$. From this fact the theorem follows easily. 
There is a converse statement to Theorem 2.1 which can be formulated in a purely geometric manner.

THEOREM 2.2. Let $H(z)=\left(B_{1}(z) \cdot \sigma_{1}+B_{2}(z) \cdot \sigma_{2}+B_{3}(z) \cdot \sigma_{3}\right)$ be a $2 \times 2$ matrix which satisfies conditions I, II, III, $\mathrm{V}^{\prime}$ on $S_{a}$. Let $\psi_{T}$ be a normalized solution of the Schroedinger equation $i \psi_{T}^{\prime}=T H \psi_{T}$ such that $\lim _{t \rightarrow-*}\left\|P_{1}(t) \psi_{T}(t)\right\|=1$. If there exists a horizontal geodesic in $S_{a}$ (in the $\rho$-geometry, $\rho=B_{1}^{2}+B_{2}^{2}+B_{3}^{2}$ ), $t \mapsto g(t)$, $t \in \mathbb{R}$, which contains exactly one eigenvalue-crossing of $H$, say $z_{1}$, and such that

$$
\lim _{t \rightarrow \pm x} \operatorname{Re} g(t)= \pm \infty
$$

and

$$
|\operatorname{Im} g(t)|<a, \quad|t| \text { large enough }
$$

then

$$
\lim _{t \rightarrow \infty}\left\|P_{2}(t) \psi_{T}(t)\right\|^{2}=\exp \left(2 \operatorname{Im} \theta_{21}(0 \mid \eta)\right) \exp \left(-2 T d_{\rho}\left(z_{1}, \mathbb{R}\right)\right)\left(1+O\left(\frac{1}{T}\right)\right)
$$

and for all eigenvalue-crossings $z_{k}, k>1, d_{\rho}\left(z_{k}, \mathbb{R}\right)>d_{\rho}\left(z_{1}, \mathbb{R}\right)$. The geometric factor $\exp \left(2 \operatorname{Im} \theta_{21}(0 \mid \eta)\right)$ is the same as in $(2.58)$.

Proof. I.et us first notice that the hypotheses imply that there is no critical point in the open set between the real axis and the geodesic $g$ (this follows, e.g., from Teichmueller's lemma). We know from the remark following Theorem 2.1 that $\Phi$ is univalent in this region and also that $d_{\rho}\left(z_{k}, \mathbb{R}\right)>d_{\rho}\left(z_{1}, \mathbb{R}\right)$ if $k>1$. The theorem is proven if we can find a path $\tilde{\gamma}$ satisfying condition VI. We assume that the parametrization of the geodesic $g$ is a natural one and that $g(0)=z_{1}$. Let $\alpha_{1}$ be the curve $t \mapsto g(t)=\alpha_{1}(t), t \in(-\infty, 0)$, and $\alpha_{2}$ be the curve $t \mapsto g(t)=\alpha_{2}(t), t \in(0, \infty)$. By hypothesis $\alpha_{1}$ and $\alpha_{2}$ are critical trajectories. Let $\alpha_{3}$ be the third critical trajectory having $z_{1}$ as accumulation point. Let $U$ be a small disc of center $z_{1}$. The three trajectories $\alpha_{1}, \alpha_{2}$, and $\alpha_{3}$ divide $U$ into three sectors as in Fig. 6. Let $z^{\prime} \in U_{1}$ and

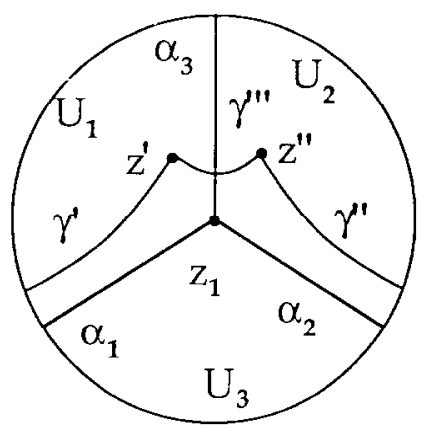

FIG. 6. The critical trajectories in $U$. 
let $u \mapsto \gamma^{\prime}(u), u \in\left(u_{-}^{\prime}, u_{+}^{\prime}\right)$ be the trajectory passing through $z^{\prime}$. We assume that the parametrization is natural and is chosen in such a way that $\gamma^{\prime}(0)=z^{\prime}$ and $\gamma^{\prime}(u)$ tends to $\alpha_{1}(u)$ when $z^{\prime}$ tends to $z_{1}$ if $u \in(-\varepsilon, 0)$ for small positive $\varepsilon$. However, since condition II holds, we have that $u_{-}^{\prime}=-\infty$ if $z^{\prime}$ is sufficiently close to $z_{1}$ and that $\gamma^{\prime}(u) \rightarrow \alpha_{1}(u)$ for all $u<0$ if $z^{\prime}$ tends to $z_{1}$. Similarly, if $z^{\prime \prime} \in U_{2}$, then the trajectory $\gamma^{\prime \prime}$ through $z^{\prime \prime}$ can be parametrized by $u \in\left(u_{-}^{\prime \prime}, \infty\right)$ in such a way that $\gamma^{\prime \prime}(0)=z^{\prime \prime}$ and $\gamma^{\prime \prime}(u)$ tends to $\alpha_{2}(u), u>0$ provided $z^{\prime \prime}$ is near $z_{1}$. Let $\gamma^{\prime \prime \prime}$ be the vertical straight arc through $z^{\prime}$ and let us choose $z^{\prime \prime}$ on $\gamma^{\prime \prime \prime}$. We can do this so that the trajectories $\gamma^{\prime}$ and $\gamma^{\prime \prime}$ have the above properties. The path $\gamma$ is defined as the composition of the path $\gamma^{\prime}$, the part of $\gamma^{\prime \prime \prime}$ between $z^{\prime}$ and $z^{\prime \prime}$, and $\gamma^{\prime \prime}$. Let $\widetilde{\Delta_{12}}$ be the analytic continuation of $A_{12}$ along $\tilde{\gamma}$, starting from some disc $V$ containing $\gamma^{\prime}(u), u<0$. If $u$ tends to $-\infty$, then we have

$$
\operatorname{Im} \tilde{\Delta_{12}}\left(\gamma^{\prime}(-\infty)\right)=\operatorname{Im} \Delta_{12}\left(\gamma^{\prime}(-\infty)\right)<\operatorname{Im} \Delta_{12}\left(\alpha_{1}(-\infty)\right) .
$$

This implies that

$$
\operatorname{Im} \tilde{\Delta_{12}}\left(z^{\prime}\right)<\operatorname{Im} \Delta_{12}\left(z_{1}\right)
$$

and

$$
\operatorname{Im} \widetilde{\Delta_{12}}\left(z^{\prime \prime}\right)>\operatorname{Im} \Delta_{12}\left(z_{1}\right)
$$

Therefore the path $\tilde{\gamma}$ has all required properties.

Remark. Let us suppose that the hypothesis of Theorem 2.2 are fulfilled except that therc is more than one eigenvalue-crossing on the horizontal geodesic $g$. Then we cannot find a dissipative path. However, we can in general treat this case by analyzing the equation along the geodesic, as in Davis and Pechukas [17].

EXAMPLE 2.1. This example illustrates the problem of selecting the relevant eigenvalue-crossing for the generalized Dykhne formula. In this example the relevant eigenvalue-crossing $i s$ not the closest one to the real axis in the Euclidean metric. This has the following consequence. We could choose the width of the analyticity strip too small so that the strip contains only the (irrelevant) closest eigenvalue-crossing to the real axis and its complex conjugate. The local analysis of [8] is still valid but it leads to an incorrect result. Let $H(z)=\mathbf{B}(z) \cdot \boldsymbol{\sigma}$ be defined by

$$
\begin{aligned}
& B_{1}(z)=2 \frac{(z-c)^{2}+b^{2} \tanh (z)}{(z-c)^{2}+a^{2}} \\
& B_{2}(z)=2 \frac{(z-c)^{2} \tanh (z)-b^{2}}{(z-c)^{2}+a^{2}} \\
& B_{3}(z) \equiv 0
\end{aligned}
$$

with $a>\frac{3}{2}, b>0$, and $c \in \mathbb{R}$. 
The singularities of $H(z)$ are located at the points $c \pm i a$ and $i \pi / 2+k i \pi, k= \pm 1$, $\pm 2, \ldots$. Hence $H(z)$ satisfies condition I in $S_{3 / 2}=\left\{z=t+i s|| s \mid<\frac{3}{2}\right\}$. One verifies that $\mathbf{B}(t+i s)$ tends to $(1,1,0)$ as $1 / t^{2}$ when $|t| \rightarrow \infty$ in $S_{3 / 2}$, so that condition II is satisfied. The function

$$
\rho(z)=4 \frac{(z-c)^{4}+b^{4}}{\left((z-c)^{2}+a^{2}\right)^{2}}\left(1+\tanh (z)^{2}\right)
$$

is strictly positive on the real axis and its zeros in $S_{3 / 2}$,

$$
z_{1}=i \frac{\pi}{4}, \quad z_{2}=c+\frac{b}{\sqrt{2}}(-1+i), \quad z_{3}=c+\frac{b}{\sqrt{2}}(1+i)
$$

and $\overline{z_{1}}, \overline{z_{2}}, \overline{z_{3}}$ are all simple. Thus condition $V^{\prime}$ is verified. We define $\Omega$ as the simply connected domain obtained from the intersection of the upper half plane and $S_{3 / 2}$, by removing three vertical cuts starting at $z_{1}, z_{2}, z_{3}$. In order to determine the relevant zero, we must study the critical trajectories $\operatorname{Im} \Delta_{12}(z)=$ $\operatorname{Im} \Delta_{12}\left(z_{j}\right), j=1,2,3$ in $\Omega$. We have computed these lines numerically for certain values of the parameters $a, b$, and $c$. For the choice $a=4, b=1.2, c=2$ we have the situation of Fig. 7 which shows that $z_{2}$ is the relevant eigenvalue-crossing and that Theorem 2.2 is true. Note that $\operatorname{Im} z_{1}=\pi / 4<1.2 / \sqrt{2}=\operatorname{Im} z_{2}$.

Remarks. (i) Condition II allows us to consider the critical trajectories in a compact subset of $\Omega$.

(ii) For the different values of the parameters we have considered, Theorem 2.2 was always true for some zero $z_{k}$.

(iii) In this example $\operatorname{Im} \theta_{21}=0$, since $B_{3} \equiv 0$ (see Appendix).

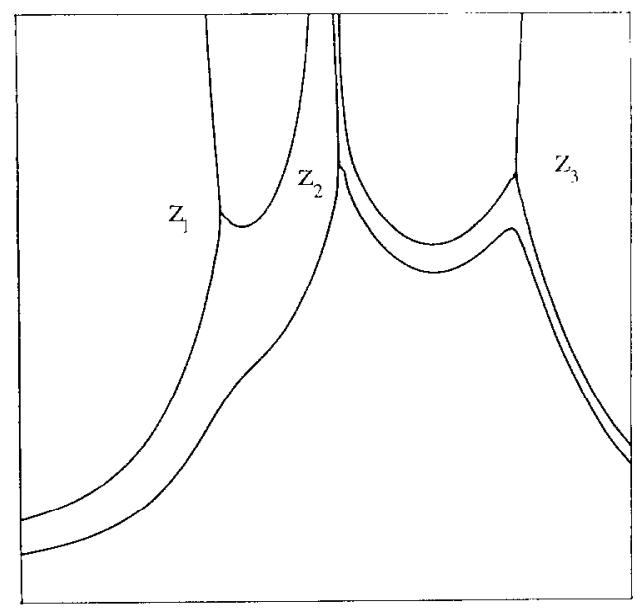

Fig. 7. The critical trajectories of example (2.1). 
EXAMPLE 2.2. In this example we have competition between one singularity of $H$ and one eigenvalue-crossing. This example is inspired by an example of [9]. The question here is again the following one. Is the probability transition determined by the singularity or the eigenvalue-crossing (see [9. Section 6])? As before it is not the fact that the singularity is closer or not to the real axis (in the Euclidean distance) than the eigenvalue-crossing which matters, but whether this is the case in the metric $d_{\rho}$ (see case (b)). Let $\mathbf{B}(z)$ be defined by

$$
\mathbf{B}(z)=2(\cos (\alpha(z)), \sin (\alpha(z)), \tanh (z)) \text { with } \alpha(z)=\frac{\pi}{4} \tanh \left(\frac{z-c}{\omega}\right),
$$

where $0<\omega<1 ; c \in \mathbb{R}$. The hamiltonian $\mathbf{B}(t+i s) \cdot \boldsymbol{\sigma}$ is singular at the point

$$
z(\omega, c)=c+i \frac{\pi \omega}{2}
$$

in the strip $S_{3 / 2}$ defined as above and tends exponentially fast to its limiting values as $|t| \rightarrow \infty$ with $|s|<3 \omega / 2$. Moreover, the function

$$
\rho(z)=4\left(1+\tanh ^{2}(z)\right)
$$

is strictly positive for $z \in \mathbb{R}$ so that conditions $I, I I$, and $V^{\prime}$ are verified. There is one pair of eigenvalue-crossings: $z_{1}=i \pi / 4$ and $\overline{z_{1}}$.

An important feature of this example is that $\rho$ is independent of $c$ and $\omega$. This implies that the $\rho$-geometry is also independent of the location of the singularity $z(\omega, c)$.

We first prove that any horizontal path $\gamma(t)$ over $z_{1}=i(\pi / 4)$ is a dissipative path. Let $\gamma(t)$ be parametrized by

$$
\gamma(t)=t+i s, \quad-\infty \leqslant t \leqslant \infty, \quad \frac{\pi}{4}<s<\frac{\pi}{2}
$$

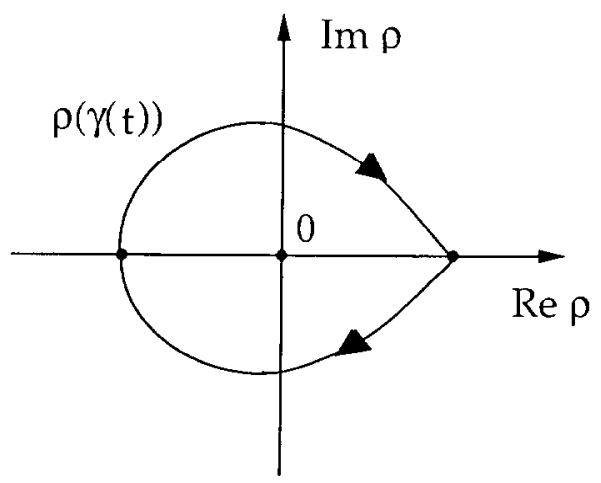

FIG. 8. The image of $\gamma$ by $\rho$. 
Such a path is dissipative if and only if

$$
\frac{d}{d t} \operatorname{Im} \Delta_{12}(\gamma(t))=-\operatorname{Im} \sqrt{\rho(\gamma(t))} \geqslant 0 \quad \forall t
$$

To see that this is true in our case, we consider the image of $\gamma(t)$ by $\rho(z)$. We compute

$$
\rho(t+i s)=4+4 \frac{\sinh ^{2}(2 t)-\sin ^{2}(2 s)}{(\cosh (2 t)+\cos (2 s))^{2}}+i \frac{8 \sinh (2 t) \sin (2 s)}{(\cosh (2 t)+\cos (2 s))^{2}}
$$

thus $\forall s \in(\pi / 4, \pi / 2)$, the image of $\gamma$ by $\rho$ looks like Fig. 8. By taking the square root, the image of $\rho(\gamma(t))$ is entirely in the lower half plane and condition (2.93) is satisfied.

Remark. The only property of the path $\rho(\gamma(t))$ which is used is that this path does not cross the positive real axis.

Since there exists a dissipative path we know that there exists a horizontal geodesic (in the $\rho$-geometry) passing through $z_{1}$ as in Theorem 2.1. A qualitative study of the differential equation satisfied by the geodesic $t \mapsto g(t)=g_{1}(t)+i g_{2}(t)$,

$$
\dot{g}_{1}(t) \operatorname{Im} \sqrt{\rho(g(t))}+\dot{g}_{2}(t) \operatorname{Re} \sqrt{\rho(g(t))}=0,
$$

shows that the critical trajectories must behave as on Fig. 9. Since

$$
\operatorname{Im} \Delta_{12}(i \pi / 4)=-2 \int_{0}^{\pi / 4} \sqrt{1-\tan ^{2}(s)} d s=-\pi(\sqrt{2}-1)
$$

$d_{p}\left(z_{1}, \mathbb{R}\right)=\pi(\sqrt{2}-1)$. We have two cases:

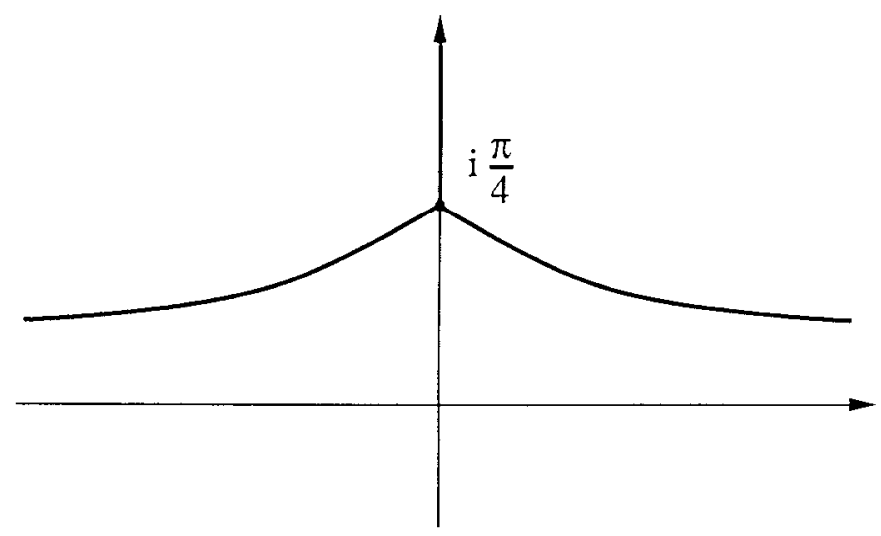

FIG. 9. The critical trajectories of example (2.2). 
(a) $\quad \omega>\frac{1}{2}$. In this case we can choose a horizontal path over the eigenvaluecrossing $z_{1}$ and below the singularity $z(\omega, c)$ of $H$. Therefore Theorem 2.2 can be applied.

(b) $\omega \leqslant \frac{1}{2}$. Here the above choice of $\gamma$ does not work because the singularity of $H$ is always below $\gamma$, since $\gamma$ must go over $z_{1}$. However, the critical trajectories are independent of $\omega$ and $c$. Hence, as long as $z(\omega, c)$ is above the critical trajectories, we can construct a dissipative path going over $z_{1}$ and below $z(\omega, c)$, as explained in the proof of Theorem 2.2, and therefore this theorem is valid. We obtain

$$
\lim _{t \rightarrow \infty}\left\|P_{2}(t) \psi_{T}(t)\right\|^{2}-\exp (-2 T \pi(\sqrt{2}-1)) \exp \left(2 \operatorname{Im} \theta_{21}\right)\left(1+O\left(\frac{1}{T}\right)\right)
$$

In this example we have a nontrivial geometric factor, $\operatorname{Im} \theta_{21}<1$, if $\omega$ is not too small. Note that in such a case.

$$
d_{p}\left(z_{1}, \mathbb{R}\right)<d_{\rho}(z(\omega, c), \mathbb{R}) .
$$

Finally, if the singularity is below the critical trajectories, we cannot prove the generalized Dykhne formula with the method described above, but we can prove upper bounds for the transition probability, as we shall see in Section 3. It will be shown that in our example the bound has the form

$$
\lim _{t \rightarrow \infty}\left\|P_{2}(t) \psi_{T}(t)\right\|^{2} \leqslant \frac{k_{\varepsilon}}{T^{2}} \exp \left(-2 T\left(d_{\rho}(z(\omega, c), \mathbb{R})-\varepsilon\right)\right) \quad \forall \varepsilon>0,
$$

where $k_{\varepsilon}$ is a constant. (Note that here we have $d_{\rho}\left(z_{1}, \mathbb{R}\right)>d_{\rho}(z(\omega, c), \mathbb{R})$.)

\section{EXPONENTIAL UPPER BOUNDS}

\subsection{Formulation of the Problem}

Let $H(z)$ be a $n \times n$ matrix defined in $S_{t}$ and which satisfies conditions I, II, and IV of the first section. From now on we suppose that the domain $S_{a}$ is a strip given by $\{z=t+i s|| s \mid<a\}$ whose width $a$ is so small that all eigenvalue-crossings are on the real axis. $\Lambda$ ccording to a theorem of Rellich [16] the eigenvalues and eigenprojections are analytic at these points because $H$ is hermitian on the real axis. We label the eigenvalues and eigenprojections as follows. Near $t=-\infty$ the spectrum of $H(t)$ is nondegenerate and if $t$ is real we label the eigenvalues in increasing order

$$
e_{1}(t)<e_{2}(t)<\cdots<e_{n}(t)
$$

$P_{j}(t)$ is the eigenprojection associated to $e_{j}(t)$. By analytic continuation we have a well-defined labelling on all $S_{a}$. As above, we construct a $z$-dependent basis of eigenvectors of $H(z)$ using the method of Section 2.1. 
Let $U(z)$ be a solution in $S_{a}$ of the equation

$$
U^{\prime}(z)=\left(\sum_{j=1}^{n} P_{j}^{\prime}(z) P_{j}(z)\right) U(z), \quad U(0)=\rrbracket .
$$

Since the eigenprojections are analytic the solution $U(z)$ is single-valued and analytic. We choose a basis of normalized eigenvectors $\varphi_{1}(0), \ldots, \varphi_{n}(0)$ of $H(0)$ and we define

$$
\varphi_{m}(z)=U(z) \varphi_{m}(0)
$$

Then we write a solution of the equation

$$
i \psi_{T}^{\prime}(z)=T H(z) \psi_{T}(z)
$$

as

$$
\psi_{T}(z)=\sum_{m=1}^{n} c_{m}(z) e^{\left.-i \lambda \lambda_{n t} t=\right)} \varphi_{m}(z),
$$

where $\lambda_{m}(z)$ is defined in (2.22). Equation (3.4) is equivalent to

$$
c_{m}^{\prime}(z)=\sum_{j \neq m}^{n} a_{m j} e^{i T 1_{m i}(z)} c_{j}(z), \quad m=1, \ldots, n
$$

with $A_{m j}(z)=\lambda_{m}(z)-\lambda_{j}(z)$. The functions $a_{m j}(z)$ are given in $(2.27)$ and, in particular, (2.28) holds, so that

$$
\lim _{t \rightarrow \pm \infty} c_{m}(t+i s)=c_{m}( \pm \infty)
$$

with limits $r_{m}( \pm \infty)$ independent of $s$. By assumption, the energy level $e_{k}$ is always isolated in the spectrum. Our problem is to estimate $c_{m}(\infty), m \neq k$, for a solution of (3.6) such that $c_{m}(-\infty)=\delta_{k m}$.

\subsection{Dissipative Paths}

The method for getting upper bounds is similar to the method used in Section 2.3. Instead of considering Eq. (3.6) on the real axis we consider complex paths in $S_{a}$. All paths below are of the type $t \mapsto \gamma(t), t \in \mathbb{R}$, with $\operatorname{Re} \gamma(t)=t$ and we put $\gamma_{2}(t)=\operatorname{Im} \gamma(t)$.

Definition. A path $\gamma$ is dissipative, for the index $j \neq k$, if $\operatorname{Im} \Delta_{k j}(\gamma(t))$ is nondecreasing.

Remarks. (i) This definition depends on the choice of the index $k$. However, $k$ is fixed by condition IV. 
(ii) A path is dissipative if and only if

$$
\operatorname{Re}\left(e_{k}(\gamma(t))-e_{j}(\gamma(t))\right) \dot{\gamma}_{2}(t)+\operatorname{Im}\left(e_{k}(\gamma(t))-e_{j}(\gamma(t))\right) \geqslant 0 .
$$

Let us suppose that $1<k<n$. If we choose the width of the band $S_{a}$ small enough, then $\operatorname{Re}\left(e_{k}(\gamma(t))-e_{j}(\gamma(t))\right)$ is strictly positive (resp. negative), when $j<k$ (resp. $j>k$ ). We assume that this is the case. Then, condition (3.8) is valid for all $j<k$ if and only if

$$
\dot{\gamma}_{2}(t) \geqslant \max _{j<k}\left(-\frac{\operatorname{Im}\left(e_{k}(\gamma(t))-e_{j}(\gamma(t))\right)}{\operatorname{Re}\left(e_{k}(\gamma(t))-e_{j}(\gamma(t))\right)}\right) .
$$

Similarly, condition (3.8) is valid for all $j>k$ if and only if

$$
\dot{\gamma}_{2}(t) \leqslant \min _{j>k}\left(-\frac{\operatorname{Im}\left(e_{k}(\gamma(t))-e_{j}(\gamma(t))\right)}{\operatorname{Re}\left(e_{k}(\gamma(t))-e_{j}(\gamma(t))\right)}\right) .
$$

In general we cannot find a path which is dissipative for all $j \neq k$ and therefore the complex adiabatic theorem of [8] is not valid. But we show in the next paragraph that there exist paths which are dissipative for all $j<k$ or all $j>k$. Note that if $n=2$ this means that there always exist dissipative paths near the real axis. The CauchyRiemann equations imply

$$
\operatorname{Im}\left(e_{k}(t+i s)-e_{j}(t+i s)\right)=\int_{0}^{s} d u \operatorname{Re}\left(e_{k}^{\prime}(t+i u)-e_{j}^{\prime}(t+i u)\right) .
$$

Therefore, we have

$$
\begin{aligned}
& \left|\operatorname{Im}\left(e_{k}(t+i s)-e_{,}(t+i s)\right)\right| \\
& \quad \leqslant|s| \sup _{|u|<a} \max _{j \neq k}\left|\operatorname{Re}\left(e_{k}^{\prime}(t+i u)-e_{j}^{\prime}(t+i u)\right)\right| \equiv|s| x(t) .
\end{aligned}
$$

Using the Cauchy formula and condition II we see that $\alpha(t)$ is an integrable function on the real line. Let

$$
C=\inf _{z \in S_{a}} \min _{j \neq k}\left|\operatorname{Re}\left(e_{k}(z)-e_{j}(z)\right)\right|
$$

and

$$
g(t, s)=\frac{\alpha(t)}{C}|s|
$$

Let us consider condition (3.10). We define a path $\gamma$ by solving the differential equation

$$
\dot{\gamma}_{2}(t)=g\left(t, \gamma_{2}(t)\right) .
$$


Thus

$$
\gamma_{2}(t)=\gamma_{2}\left(t_{0}\right) \exp \left(\int_{t_{11}}^{t} d u \frac{\alpha(u)}{C}\right), \quad \gamma_{2}\left(t_{0}\right)>0
$$

and

$$
\gamma_{2}(t)=\gamma_{2}\left(t_{0}\right) \exp \left(-\int_{t_{10}}^{t} d u \frac{\alpha(u)}{C}\right), \quad \gamma_{2}\left(t_{0}\right)<0 .
$$

By suitably choosing the initial condition $\gamma_{2}\left(t_{0}\right)$ we can require that $\left|\gamma_{2}(t)\right|<a$ so that the path is in $S_{a}$ for all $t$ and it is dissipative for all $j<k$ because of (3.9). Similarly, we define a dissipative path $\gamma$ for all $j>k$ by solving the differential equation

$$
\dot{\gamma}_{2}(t)=-g\left(t, \gamma_{2}(t)\right)
$$

Let $\eta$ be the dissipative path in $S_{u}$ for $j>k$ such that

$$
\eta_{2}(t)=\eta_{2}(\propto) \exp \left(\int_{t}^{\infty} d u \frac{\alpha(u)}{C}\right)
$$

with $\eta_{2}(\infty)>0$ as large as possible. Let $\tilde{\eta}$ be the dissipative path for $j<k$ in $S_{u}$ with

$$
\tilde{\eta}_{2}(t)=\eta_{2}(\infty) \exp \left(-\int_{t}^{\infty} d u \frac{\alpha(u)}{C}\right) .
$$

We define $D^{+}$as the closed subset of $S_{a}$ between $\eta$ and $\tilde{\eta}$ and $D^{-}$as the complex conjugate of $D^{+}$(see Fig. 10). The main property of $D^{+}$which we use below is that for any $z \in D^{+}$we can find two dissipative paths. There exists a dissipative path $\gamma$ for $j>k$ with $\gamma(t) \in D^{+}$for $t \leqslant \operatorname{Re} z$ and $\gamma(\operatorname{Re} z)=z$ and there exists a dissipative path $\tilde{\gamma}$ for $j<k$ with $\tilde{\gamma}(t) \in D^{+}$for $t \leqslant \operatorname{Re} z$ and $\tilde{\gamma}(\operatorname{Re} z)=z$ (see Fig. 10).

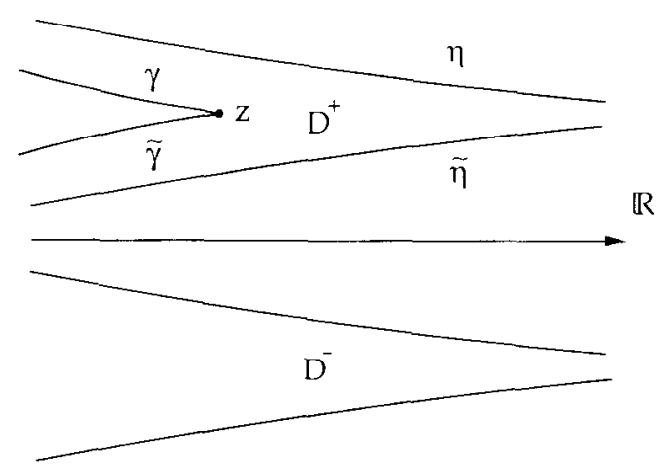

FIG. 10. The two subsets $D^{+}$and $D^{-}$. 


\subsection{Upper Bounds for Transition Probabilities}

Let $c_{j}(z), j=1, \ldots, n$, be solutions of Eqs. (3.6) with boundary conditions $\lim _{t \rightarrow-x} c_{j}(t)=\delta_{k j}$. We prove upper bounds for $\left|c_{m}(\infty)\right|, m \neq k$. Since we have property (3.7) we can consider Eqs. (3.6) on $D^{+} \cup D$. We introduce new variables

$$
x_{j}(z)=e^{i T \cdot J_{j j}(z)} c_{j}(z)
$$

$\left(x_{k}=c_{k}\right)$ and we show below that there exists a constant $M$ such that for $T$ large enough

$$
\sup _{z \in D^{+} \cup D}\left|x_{m}(z)\right| \leqslant \frac{M}{T}, \quad m \neq k .
$$

From this result we immediately get the estimate (1.11), which is the main result of this section

$$
\begin{aligned}
\lim _{t \rightarrow \infty}\left\|P_{m}(t) \psi_{T}(t)\right\|^{2} & =\left|c_{m}(\infty)\right|^{2} \\
& \leqslant \frac{M^{2}}{T^{2}} \exp \left(-2 T\left|\operatorname{Im} \Delta_{k m}(\eta(\infty))\right|\right) \\
& =\frac{M^{2}}{T^{2}} \exp \left(-2 T \eta_{2}(\infty)\left|e_{k}(\infty)-e_{m}(\infty)\right|\right)
\end{aligned}
$$

with $\eta_{2}(\infty)>0$ (see (3.19)). We prove the bound (3.22) separately on $D^{+}$and on $D^{-}$. Let us consider a point $z \in D^{+}$and the two paths $\gamma$ and $\tilde{\gamma}$ defined in the preceding section. We use the notations $c_{m}(t) \equiv c_{m}(\gamma(t)), a_{m j}(t) \equiv a_{m j}(\gamma(t))$, etc. Along the path $\gamma$, Eq. (3.6) for $c_{m}$ reads

$$
c_{m}(t)=\delta_{k m}+\sum_{j \neq m}^{n} \int_{-\infty}^{t} d u \dot{\gamma}(u) a_{m j}(u) e^{i T A_{m l}(u)} c_{j}(u) .
$$

We perform an integration by parts,

$$
\begin{aligned}
c_{m}(t)= & \delta_{k m}+\sum_{i \neq m}^{n} \frac{1}{i T} \frac{a_{m j}(t)}{A_{m j}^{\prime}(t)} e^{i T \Delta_{m j}(t)} c_{j}(t) \\
& -\sum_{i \neq m}^{n} \frac{1}{i T} \int_{-\infty}^{t} d u \dot{\gamma}(u)\left(\frac{a_{m j}}{\Delta_{m i}^{\prime}}\right)^{\prime}(u) e^{i T \Delta_{m i}(u)} c_{j}(u) \\
& -\sum_{j \neq m}^{n} \frac{1}{i T} \sum_{r \neq j}^{n} \int_{\infty}^{t} d u \dot{\gamma}(u) \frac{a_{m j} a_{j r}}{\Delta_{m j}^{\prime}}(u) e^{i T \Delta_{m r}(u)} c_{r}(u)
\end{aligned}
$$


and we rewrite the result (3.25) using the variables $x_{m}(t) \equiv x_{m}(\gamma(t))$. After multiplication by $e^{i T \Delta_{k m}(t)}$ if $m \neq k$, we obtain

$$
\begin{aligned}
x_{m}(t)= & \delta_{k m}+\sum_{j \neq m}^{n} \frac{1}{i T} \frac{a_{m j}(t)}{\Delta_{m j}^{\prime}(t)} x_{j}(t) \\
& -\sum_{j \neq m}^{n} \frac{1}{i T} \int_{\varkappa}^{t} d u \dot{\gamma}(u)\left(\frac{a_{m j}}{\Delta_{m j}^{\prime}}\right)^{\prime}(u) e^{i T\left(\Delta_{k m}(t)-\Delta_{k m}(u) x_{j}\right.} x_{j}(u) \\
& -\sum_{j \neq m}^{n} \frac{1}{i T} \sum_{r \neq j}^{n} \int_{-\varsigma}^{t} d u \dot{\gamma}(u) \frac{a_{m j} a_{j r}}{A_{m j}^{\prime}}(u) e^{i T\left(\Delta_{k m}(t)-\Delta_{k m}(u) \prime\right.} x_{r}(u) .
\end{aligned}
$$

Let $\left\|x_{j}\right\|=\sup _{z \in D^{+}}\left|x_{j}(z)\right|$. Since the path $\gamma$ is dissipative for $m>k$ we have for every $m \geqslant k$

$$
\left|e^{i T\left(A_{k m}(t)-\Delta_{k m(t)}(t)\right.}\right| \leqslant 1, \quad t \geqslant u
$$

and therefore we obtain for $m \geqslant k$

$$
\left|x_{m}(\gamma(\operatorname{Re} z))\right| \leqslant \delta_{k m}+\frac{M^{\prime}}{T}\left(\sum_{i=1}^{n}\left\|x_{j}\right\|\right) .
$$

Similarly, if we use the path $\tilde{\gamma}$ which is dissipative for $m<k$, we obtain (3.28) for $m<k$. The constant $M^{\prime}$ in (3.28) can be chosen independently of $z$. Therefore we take the supremum over $z \in D^{+}$in (3.28) and we obtain

$$
\left\|x_{m}\right\| \leqslant \delta_{k m}+\frac{M^{\prime}}{T}\left(\sum_{j=1}^{n}\left\|x_{j}\right\|\right) .
$$

If $T$ is large enough

$$
\sum_{j=1}^{n}\left\|x_{j}\right\| \leqslant \frac{1}{2}
$$

and thus

$$
\left\|x_{m}\right\| \leqslant \frac{M^{\prime}}{2 T}, \quad m \neq k
$$

The bound for $z \in D^{-}$is proven in the same manner.

Remarks. (i) If $k=1$ or $k=n$ the proof is simpler since we can work only with one single path above or below the real axis. The integration by parts is not necessary. If we do not perform it, then we obtain

$$
\lim _{t \rightarrow \infty}\left\|P_{m}(t) \psi_{T}(t)\right\|^{2} \leqslant \text { Const } \exp \left(-2 T \eta_{2}(\infty)\left|e_{k}(\infty)-e_{m}(\infty)\right|\right) .
$$

(ii) If $n=2$ we can construct dissipative paths as follows. Let $S_{a}$ be a simply connected domain where the analytic function $z \mapsto \Delta_{12}(z)$ is univalent, i.e., one-to- 
one. Let $A_{a}$ be the image of $S_{a}$ by $A_{12}$. In $A_{a}$ we choose the horizontal path which is in the upper half-plane and as distant as possible from the real axis. (We are discussing the case where $\lim _{t \rightarrow-x}\left\|P_{1}(t) \psi_{T}(t)\right\|=1$ and $e_{1}(t)$ is the lowest energy level.) We take as the dissipative path $\gamma$ the inverse image of this horizontal path. Note that $\left|e_{1}(\infty)-e_{2}(\infty)\right| \operatorname{Im} \gamma(\infty)$ is the Euclidean distance of the horizontal line $\Delta_{12}(\gamma)$ in $A_{a}$ to the real axis. Let us suppose that the hypotheses of Theorem 2.2 are verified. Then we can take for $S_{a}$ the domain between the geodesics $g$ through $z_{1}$ and $\bar{g}$ through $\overline{z_{1}}$. Its image by $\Lambda_{12}$ is a strip $\Lambda_{a}$ of width $2 d_{p}\left(z_{1}, \mathbb{R}\right.$ ) (in the Euclidean distance). From this follows (1.12) (see the remark after Theorem 2.1).

\section{A. ApPendiX}

We derive in this appendix an explicit formula in terms of the components $B_{j}$ for the phase $\theta_{21}(0 \mid \gamma)$ defined by

$$
U(0 \mid \gamma) \varphi_{1}(0)=e^{-i\left(\theta_{21}(0+\gamma)\right.} \varphi_{2}(0)
$$

In order to do that we consider the set of analytic eigenvectors

$$
\psi_{j}(z)=\left(B_{3}(z)+(-1)^{\prime} \sqrt{\rho(z)}, B_{1}(z)+i B_{2}(z)\right), \quad j=1,2,
$$

associated to the eigenvalues

$$
e_{j}(z)=(-1)^{j} \frac{1}{2} \sqrt{\rho(z)}, \quad j=1,2 .
$$

Writing $\psi_{j}(0 \mid \gamma)$ the analytic continuations of $\psi_{j}(z)$ along $\gamma$, we have

$$
\psi_{j}(0 \mid \gamma)=\psi_{k}(0) \quad \text { with } j \neq k \text {. }
$$

We define the new analytic phases $\delta_{j}(z)$ by the relation

$$
\psi_{j}(z)=e^{\left.i s_{j} t z\right)} \varphi_{j}(z)
$$

and we have

$$
\psi_{j}^{\prime}(z)=i \delta_{j}^{\prime}(z) \psi_{j}(z)+e^{i \delta j(z)} \varphi_{j}^{\prime}(z)
$$

Applying the projection $P_{j}(z)$ to this identity and using property $(2.16)$ we obtain the relation

$$
P_{i}(z) \psi_{j}^{\prime}(z)=i \delta_{j}^{\prime}(z) \psi_{j}(z)
$$

and by taking the scalar product with $\psi_{j}(z)$

$$
i \delta_{j}^{\prime}(z)=\frac{\left\langle\psi_{j}(z) \mid P_{j}(z) \psi_{j}^{\prime}(z)\right\rangle}{\left\langle\psi_{j}(z) \mid \psi_{j}(z)\right\rangle} .
$$


On the real axis, $P_{j}(z)$ is self-adjoint, so that

$$
i \delta_{j}^{\prime}(x)=\frac{\left\langle\psi_{j}(x) \mid \psi_{j}^{\prime}(x)\right\rangle}{\left\langle\psi_{i}(x) \mid \psi_{j}(x)\right\rangle} \quad \text { for } \quad x \in \mathbb{R} .
$$

As $(d / d x)\left\langle\psi_{j}(x) \mid \psi_{j}(x)\right\rangle=2 \operatorname{Re}\left\langle\psi_{j}(x) \mid \psi_{j}^{\prime}(x)\right\rangle$ we can write

$$
i \delta_{j}^{\prime}(x)=\frac{1}{2} \frac{d}{d x} \ln \left\langle\psi_{j}(x) \mid \psi_{j}(x)\right\rangle+i \operatorname{Im} \frac{\left\langle\psi_{j}(x) \mid \psi_{j}^{\prime}(x)\right\rangle}{\left\langle\psi_{j}(x) \mid \psi_{j}(x)\right\rangle} .
$$

From the expression (A.2) we compute

$$
\left\langle\psi_{j}(x) \mid \psi_{j}(x)\right\rangle=2 \sqrt{\rho(x)}\left(\sqrt{\rho(x)}+(-1)^{j} B_{3}(x)\right)
$$

and

$$
\operatorname{Im}\left\langle\psi_{j}(x) \mid \psi_{j}^{\prime}(x)\right\rangle=B_{1}(x) B_{2}^{\prime}(x)-B_{2}(x) B_{1}^{\prime}(x)
$$

Since both expressions possess an analytic continuation obtained by putting $z \in \mathbb{C}$ in place of $x \in \mathbb{R}$ we eventually obtain

$$
\begin{aligned}
i \delta_{j}^{\prime}(z)= & \frac{1}{2} \frac{d}{d z} \ln \left(2 \sqrt{\rho(z)}\left(\sqrt{\rho(z)}+(-1)^{j} B_{3}(z)\right)\right) \\
& +i \frac{B_{1}(z) B_{2}^{\prime}(z)-B_{2}(z) B_{1}^{\prime}(z)}{2 \sqrt{\rho(z)}\left(\sqrt{\rho(z)}+(-1)^{j} B_{3}(z)\right)} \\
= & \frac{1}{2} \frac{d}{d z} \ln \left(2 \sqrt{\rho(z)}\left(\sqrt{\rho(z)}+(-1)^{j} B_{3}(z)\right)\right)+i \frac{d}{d z} \ln \left(\frac{B_{1}(z)-i B_{2}(z)}{B_{1}(z)+i B_{2}(z)}\right) \\
& -i(-1)^{j} \frac{B_{3}(z)\left(B_{1}(z) B_{2}^{\prime}(z)-B_{2}(z) B_{1}^{\prime}(z)\right)}{2 \sqrt{\rho(z)}\left(B_{1}^{2}(z)+B_{2}^{2}(z)\right)} .
\end{aligned}
$$

The definition (A.5) and property (A.4) imply the relation

$$
\varphi_{1}(0 \mid \gamma)=\exp \left(-i \delta_{1}(0)-i \int_{\vartheta} \delta_{1}^{\prime}(z) d z+i \delta_{2}(0)\right) \varphi_{2}(0)
$$

with $\delta_{j}(0)$ given by the choice

$$
\psi_{j}(0)=\left\|\psi_{j}(0)\right\| \varphi_{j}(0)=e^{i i_{j}(0)} \varphi_{j}(0) .
$$

Using (A.13) and (A.15) we obtain for the exponent in (A.14)

$$
\begin{aligned}
-i \int_{\gamma} & \frac{B_{3}\left(B_{1} B_{2}^{\prime}-B_{2} B_{1}^{\prime}\right)}{2 \sqrt{\rho}\left(B_{1}^{2}+B_{2}^{2}\right)}(z) d z-i \int_{\gamma} \frac{d}{d z} \ln \left(\frac{B_{1}-i B_{2}}{B_{1}+i B_{2}}\right)(z) d z \\
& -\left.\frac{i}{2} \arg 2 \sqrt{\rho}\left(\sqrt{\rho}-B_{3}\right)(z)\right|_{0} ^{|0|} .
\end{aligned}
$$


Here $(0 \mid \gamma)$ denotes the endpoint of $\gamma$. We have assumed a square root branch point for $\sqrt{\rho}$ at the eigenvalue-crossing $z_{1}$ so that $B_{1}\left(z_{1}\right) \pm i B_{2}\left(z_{1}\right) \neq 0$ and $\sqrt{\rho\left(z_{1}\right)} \pm$ $B_{3}\left(z_{1}\right) \neq 0$. Hence we can choose a path $\gamma$ encircling neither singularities nor zeros of $\left(B_{1}-i B_{2}\right) /\left(B_{1}+i B_{2}\right)$ and such that $\left.\arg 2 \sqrt{\rho}\left(\sqrt{\rho}-B_{3}\right)(z)\right|_{0} ^{(0 \mid \eta)}=-\pi$. We obtain the following expression for $\theta_{21}(0 \mid \gamma)$ :

$$
\exp \left(-i \theta_{21}(0 \mid \gamma)\right)=\exp \left(i \frac{\pi}{2}-i \int_{\gamma} \frac{B_{3}(z)\left(B_{1}(z) B_{2}^{\prime}(z)-B_{2}(z) B_{1}^{\prime}(z)\right)}{2 \sqrt{\rho(z)}\left(B_{1}^{2}(z)+B_{2}^{2}(z)\right)} d z\right)
$$

Note added in proof. We learn from the referee that the geometrical factor in (1.6) found by Berry [9] and us has already led to experimental confirmation. We thank the referee for this information.

\section{RFFERENCES}

1. M. Born and V. Fock, Beweis des Adiabatensatzes, Z. Phys. 51 (1928), 165-180.

2. M. V. Berry, Quantal phase factor accompanying adiabatic changes, Proc. Roy. Soc. London A 392 (1984), 45-57.

3. A. Shapere and F. Wilczek, "Geometric Phases in Physics," World Scientific, Singapore/ New Jersey/London/Hong Kong, 1989.

4. B. Simon, Holonomy, the quantum adiabatic theorem, and Berry's phase, Phys. Rev. Lett. 51 (1983), $2167-2170$.

5. E. A. Solov'ev, Nonadiabatic transitions in atomic collisions, Sov. Phys. Usp. 32 (1989), 228-250.

6. A. M. DykhNe, Adiabatic perturbation of discrete spectrum states, Sot. Phys. JETP 14 (1962), 941-943.

7. L. D. Landau and E. M. Lifshitz, "Quantum Mechanics," Pergamon, Elmsford, NY, 1965.

8. J.-T. Hwang and P. PeChukas, The adiabatic theorem in the complex plane and the semi-classical calculation of non-adiabatic transition amplitudes, J. Chem. Phys. 67 (1977), 4640-4653.

9. M. V. Berry, Geometric amplitude factors in adiabatic quantum transitions, Proc. Roy. Soc. London A 430 (1990), 405-411.

10. A. Joye, H. Klnz, and Ch.-Ed. Pfister, Exponential decay and lopological aspects of nonadiabatic transition probabilities, EPFL preprint, 1989.

11. A. Joyf and Ch.-Ed. Pfister, Exponentially small adiabatic invariant for the Schroedinger equation, Commum. Math. Phys., to appear.

12. T. Kato, "Perturbation Theory for Linear Operators," Springer-Verlag, Berlin/Heidelberg/ New York, 1966.

13. K. Strebel, "Quadratic Differentials." Springer-Verlag, Berlin/Heidelberg, 1984.

14. M. Fedoriouk, "Méthodes Asymptotiques pour les Equations Différentielles Ordinaires Linéaires," MIR, Moscow, 1987.

15. O. Lehto, "Univalent Functions and Teichmueller Spaces," Springer-Verlag, Berlin/Heidelberg, 1986.

16. F. Rellich, "Perturbation Theory of Eigenvalue Problems," Gordon \& Breach Science, New York/ London/Paris, 1969.

17. J. Davis and P. Pechukas, Nonadiabatic transitions induced by a time-dependent Hamiltonian in the semiclassical/adiabatic limit: The two-state case, J. Chem. Phys. 64 (1976), 3129-3137. 